\title{
26. DATA REPORT: GEOCHEMISTRY AND MINERAL CHEMISTRY OF ULTRAMAFIC ROCKS FROM THE KANE AREA (MARK) ${ }^{1}$
}

\author{
Carl-Dietrich Werner ${ }^{2}$ and Joachim Pilot $^{2}$
}

\begin{abstract}
A suite of serpentinized peridotites from Leg 153 was analyzed for major and trace elements. All the rocks are less-refractory peridotites. The mineral chemistry of olivines $(\mathrm{Mg} \#=90.3)$, orthopyroxenes $(\mathrm{Mg} \#=90.4)$, clinopyroxenes $(\mathrm{Mg} \#=91.6)$, and some $\mathrm{Cr}$ spinels $(\mathrm{Mg} \#=68.4 ; \mathrm{Cr} \#=29.7)$ shows the characteristic features of minerals from less-depleted mantle peridotites. Some geothermometric calculations gave temperatures for the pyroxene system of about $1300^{\circ} \mathrm{C}$, reflecting the prekinematic to synkinematic crystallization of the pyroxenes, and gave a range from $1180^{\circ}$ to $1075^{\circ} \mathrm{C}$ for the mineral pair orthopyroxene and spinel. Oxygen-isotope determinations show $\delta^{18} \mathrm{O}$ values of about $6 \%$ for olivine, orthopyroxene, and clinopyroxene; $\delta^{18} \mathrm{O}$ values from chromian spinel/magnetite vary between $2.9 \%$ and $3.8 \%$, values from serpentine vary between $2.8 \%$ and $5.3 \%$, and whole-rock values vary between $2.9 \%$ and $4.6 \%$. Two temperatures could be calculated from the oxygenisotope exchange for the mineral pair clinopyroxene and spinel: about $1100^{\circ}$ and about $900^{\circ} \mathrm{C}$. The $1100^{\circ} \mathrm{C}$ value nearly corresponds to the data obtained using the orthopyroxene-spinel mineral thermometer, and may indicate subsolidus deformation and recrystallization. The temperature range of the serpentinization could only be estimated. It depends upon the rock/water ratio, and may not have exceeded $\sim 300^{\circ} \mathrm{C}$. Finally, a calculation of possible rock/water ratios is given.
\end{abstract}

\section{INTRODUCTION}

Serpentinized peridotites are exposed in a belt about $2 \mathrm{~km}$ wide and more than $20 \mathrm{~km}$ long on the western median valley wall of the Mid-Atlantic Ridge south of the Kane Fracture Zone (MARK) at $23^{\circ} 20^{\prime} \mathrm{N}$. This outcrop is interpreted as a part of the oceanic upper mantle uplifted during tectonic extension of the slowly spreading Mid-Atlantic Ridge (Cannat et al., 1995; Karson and Winters, 1992; Purdy and Detrick, 1986).

Shipboard documentation has shown that the ultramafic complex from Site 920 (Leg 153) can be divided into several "units," which differ especially in their fabrics and slightly in their degrees of serpentinization. All samples are moderately to strongly serpentinized harzburgites with $15 \%-30 \%$ relict minerals (Cannat, Karson, Miller, et al., 1995).

Polished thin sections were used for microscope investigations and also for microprobe work, which was conducted on the "CAMEBAX S 50" at the Ruhr Universität, Bochum.

Major-element oxides and trace elements $(\mathrm{Ga}, \mathrm{Pb}, \mathrm{Cu}, \mathrm{Zn}, \mathrm{Y}, \mathrm{Nb}$, $\mathrm{Zr}, \mathrm{Ti}, \mathrm{V}, \mathrm{Ni}, \mathrm{Co}, \mathrm{Cr}, \mathrm{Sc}, \mathrm{S}$, and $\mathrm{Cl}$ ) were measured by $\mathrm{X}$-ray fluorescence spectroscopy (XRF) using a Philips PW 1480 sequential spectrometer. The XRF was used to analyze both pressed pellets and glass discs prepared from powdered, but not ignited, material with lithium tetraborate. $\mathrm{Na}, \mathrm{K}, \mathrm{Li}, \mathrm{Rb}$, and $\mathrm{Zn}$ were analyzed by flame photometry, $\mathrm{Sr}$ and $\mathrm{Ba}$ by inductively coupled plasma-atomic emission spectroscopy (ICP-AAS) using a Perkin-Elmer PLASMA 1000 spectrometer, and As and Sb by inductively coupled plasma-mass spectrometry (ICP-MS) using a Perkin-Elmer ELAN 5000 spectrometer. Boron was determined by optical spectroscopy, with boron-free spectral carbons, using a Carl Zeiss PGS 2 spectrograph.

$\delta^{18} \mathrm{O}$ was determined from whole-rock samples and from mineral separates of olivine, orthopyroxene, clinopyroxene, serpentine, and chromian spinel/magnetite.

'Karson, J.A., Cannat, M., Miller, D.J., and Elthon, D. (Eds.), 1997. Proc. ODP, Sci. Results, 153: College Station, TX (Ocean Drilling Program).

${ }^{2}$ TU Bergakademie Freiberg, Institut für Mineralogie, D-09596 Freiberg, Federal Republic of Germany.

\section{SAMPLE MATERIAL}

Two samples from Hole 920B and 8 samples from Hole 920D, together with an Ocean Drilling Program (ODP) internal laboratory standard ("Ultra"), were chosen for geochemical investigation. Two further samples from Hole 920D were used only for microprobe investigations. The special sample designations correspond to the shipboard documentation (Cannat, Karson, Miller, et al., 1995), but simpler numbers, defined in Table 1 , are used in most of the tables and parts of the text.

\section{WHOLE-ROCK GEOCHEMISTRY}

The results of the chemical investigations are compiled in Table 2. The CIPW (after the authors Cross, Iddings, Pearson, Washington) norm calculations (converted to $100 \%$ and with total $\mathrm{Fe}$ as $\mathrm{Fe}^{2+}$ ) are listed in Table 3. Generally, the chemical and mineralogical compositions of the investigated ultramafic rocks are within the ranges previously reported for the Kane region by Aumento and Loubat (1971), Cannat et al. (1992), Dick et al. (1984), Michael and Bonatti (1985), and Miyashiro et al. (1969). The chemical and mineralogical data show the characteristic features of serpentinized harzburgitic ultra-

Table 1. Identification of sample numbers.

\begin{tabular}{clr}
\hline $\begin{array}{c}\text { Sample } \\
\text { number }\end{array}$ & Hole, core, section, piece & $\begin{array}{r}\text { Depth } \\
\text { (mbsf) }\end{array}$ \\
\hline 1 & 920B-8R-4, Piece 11 & 75.5 \\
2 & 902B-13R-1, Piece 2C & 117.6 \\
5 & 920D-3R-1, Piece 6 & 18.2 \\
6 & 920D-5R-1, Piece 4 & 37.1 \\
8 & 920D-14R-4, Piece 6 & 119.4 \\
10 & 920D-16R-1, Piece 5D & 134.4 \\
11 & 920D-16R-6, Piece 10 & 140.3 \\
12 & 920D-19R-1, Piece 5 & 162.7 \\
13 & 920D-20R-5, Piece 4 & 177.6 \\
14 & 920D-21R-2, Piece 5B & 183.9 \\
15 & 920D-22R-2, Piece 1D & 192.1 \\
16 & 920D-22R-4, Piece 1B & 193.7 \\
\hline
\end{tabular}


Table 2. Chemical composition of ultramafic rocks from Leg 153.

\begin{tabular}{|c|c|c|c|c|c|c|c|c|c|c|c|}
\hline Sample no: & 1 & 3 & 5 & 6 & 8 & 10 & 12 & 13 & 14 & 16 & \\
\hline Hole: & $920 \mathrm{~B}$ & $920 \mathrm{~B}$ & $920 \mathrm{D}$ & $920 \mathrm{D}$ & 920D & $920 \mathrm{D}$ & $920 \mathrm{D}$ & $920 \mathrm{D}$ & $920 \mathrm{D}$ & $920 \mathrm{D}$ & \\
\hline Core: & $8 \mathrm{R}$ & $13 R$ & $3 R$ & $5 R$ & $14 \mathrm{R}$ & $16 \mathrm{R}$ & $19 \mathrm{R}$ & $20 \mathrm{R}$ & $21 \mathrm{R}$ & $22 \mathrm{R}$ & Ultra \\
\hline Section, piece: & 4,11 & $1,2 \mathrm{C}$ & 1,6 & 1,4 & 4,6 & $1,5 \mathrm{D}$ & 1,5 & 5,4 & $2,5 \mathrm{~B}$ & $4,1 \mathrm{~B}$ & \\
\hline Depth (mbsf): & 75.5 & 117.6 & 18.2 & 37.1 & 119.4 & 134.4 & 162.7 & 177.6 & 183.9 & 193.7 & \\
\hline \multicolumn{12}{|c|}{ Major elements (wt\%) } \\
\hline $\mathrm{SiO}_{2}$ & 39.5 & 38.15 & 38.7 & 37.9 & 39.3 & 38.3 & 39.0 & 39.2 & 38.1 & 39.4 & 38.8 \\
\hline $\mathrm{TiO}_{2}$ & 0.02 & 0.011 & 0.02 & 0.018 & 0.008 & 0.013 & 0.014 & 0.024 & 0.02 & 0.008 & 0.02 \\
\hline $\mathrm{Al}_{2} \mathrm{O}_{3}$ & 1.13 & 1.07 & 1.2 & 1.21 & 1.3 & 1.12 & 1.26 & 1.31 & 1.15 & 1.2 & 1.18 \\
\hline $\mathrm{Cr}_{2} \mathrm{O}_{3}$ & 0.34 & 0.35 & 0.34 & 0.39 & 0.38 & 0.36 & 0.39 & 0.37 & 0.37 & 0.41 & 0.365 \\
\hline $\mathrm{Fe}_{2} \mathrm{O}_{3}$ & 7.5 & 7.92 & 8.15 & 7.92 & 8.31 & 8.09 & 8.26 & 7.93 & 7.95 & 8.04 & 8.34 \\
\hline $\mathrm{MnO}$ & 0.119 & 0.112 & 0.087 & 0.12 & 0.12 & 0.11 & 0.124 & 0.113 & 0.12 & 0.122 & 0.103 \\
\hline $\mathrm{MgO}$ & 38.4 & 38.8 & 38.5 & 38.9 & 38.65 & 38.7 & 37.7 & 37.8 & 38.8 & 38.8 & 37.6 \\
\hline $\mathrm{NiO}$ & 0.27 & 0.27 & 0.27 & 0.27 & 0.28 & 0.29 & 0.29 & 0.27 & 0.28 & 0.27 & 0.286 \\
\hline $\mathrm{CaO}$ & 0.24 & 0.69 & 0.14 & 0.06 & 0.94 & 0.83 & 0.30 & 0.65 & 0.35 & 1.18 & 0.13 \\
\hline $\mathrm{Na}_{2} \mathrm{O}$ & 0.09 & 0.056 & 0.06 & 0.06 & 0.05 & 0.09 & 0.07 & 0.08 & 0.07 & 0.08 & 0.045 \\
\hline $\mathrm{K}_{2} \mathrm{O}$ & 0.027 & 0.009 & 0.032 & 0.018 & 0,020 & 0.009 & 0.004 & 0.012 & 0.012 & 0.009 & 0.004 \\
\hline $\mathrm{P}_{2} \mathrm{O}_{5}$ & 0.011 & 0.010 & 0.010 & 0.011 & 0.011 & 0.011 & 0.007 & 0.012 & 0.010 & 0.010 & 0.005 \\
\hline LOI & 12.3 & 12.8 & 12.5 & 13.0 & 10.5 & 12.2 & 12.5 & 12.6 & 12.7 & 10.7 & 13.0 \\
\hline Total & 99.95 & 100.25 & 100.01 & 99.88 & 99.87 & 100.12 & 99.92 & 100.37 & 99.93 & 100.23 & 99.82 \\
\hline \multicolumn{12}{|c|}{ Trace elements (ppm) } \\
\hline B & 25 & 10 & 24 & 21 & 9 & 5 & 21 & 8 & 24 & 9 & 18 \\
\hline $\mathrm{Li}$ & 5 & 1 & - & - & 1 & - & 1 & 1 & 2 & 2 & \\
\hline $\mathrm{Rb}$ & $<1$ & - & - & - & - & - & - & $<1$ & - & $\sim 1$ & - \\
\hline $\mathrm{Sr}$ & 2.85 & 2.89 & 2.47 & 0.93 & 1.45 & 1.21 & 4.65 & 2.28 & 2.59 & 2.47 & 2.24 \\
\hline $\mathrm{Ba}$ & 0.31 & 1.62 & 0.70 & 0.53 & 1.25 & 0.43 & 0.74 & 1.01 & 0.98 & 0.95 & 1.9 \\
\hline $\mathrm{Ga}$ & - & $<1$ & $<2$ & $<2$ & - & - & $<1$ & $\overline{0}$ & $<2$ & $\overline{-}$ & - \\
\hline $\mathrm{Pb}$ & $\sim 3$ & 4 & 4 & - & 6 & $\sim 2$ & 5 & $<2$ & 7 & $\sim 2$ & $<3$ \\
\hline $\mathrm{Cu}$ & 16 & 17 & 24 & 43 & 19 & 27 & 15 & 17 & 21 & 22 & 12 \\
\hline $\mathrm{Zn}$ & 36 & 38 & 36 & 54 & 47 & 42 & 40 & 37 & 44 & 46 & 40 \\
\hline $\mathrm{Y}$ & -1 & $<1$ & $<1$ & - & $\sim 1$ & $\sim 1$ & $<1$ & - & $<1$ & - & - \\
\hline $\mathrm{Nb}$ & - & - & $\sim 1$ & $<1$ & $<1$ & - & $<1$ & $<1$ & $<1$ & $\overline{-}$ & $<1$ \\
\hline $\mathrm{Zr}$ & 4 & 3 & -3 & 3 & 4 & $\sim 3$ & 4 & 4 & 4 & 5 & 3 \\
\hline $\mathrm{Ti}$ & 120 & 65 & 120 & 110 & 50 & 80 & 85 & 145 & 120 & 50 & 120 \\
\hline V & 46 & 44 & 47 & 48 & 48 & 43 & 48 & 49 & 51 & 48 & 50 \\
\hline $\mathrm{Ni}$ & 2150 & 2135 & 2105 & 2130 & 2180 & 2250 & 2250 & 2145 & 2205 & 2150 & 2250 \\
\hline Co & 102 & 101 & 98 & 102 & 110 & 108 & 112 & 101 & 99 & 99 & 109 \\
\hline $\mathrm{Cr}$ & 2350 & 2410 & 2335 & 2690 & 2620 & 2490 & 2690 & 2500 & 2550 & 2780 & 2500 \\
\hline $\mathrm{Sc}$ & 14 & 10 & 11 & 11 & 14 & 11 & 12 & 12 & 8 & 9 & 11 \\
\hline $\mathrm{S}$ & 865 & 525 & 890 & 870 & 425 & 540 & 1050 & 885 & 770 & 380 & 990 \\
\hline As & 0.22 & 0.71 & 0.42 & 0.40 & 1.38 & 0.21 & 2.0 & 2.3 & 0.42 & 1.18 & 0.71 \\
\hline Sb & 0.24 & 0.25 & 0.32 & 0.07 & 0.34 & - & 0.04 & 0.40 & 0.52 & 0.22 & $\bar{x}$ \\
\hline $\mathrm{Cl}$ & 1095 & 3795 & 855 & 830 & 2210 & 2310 & 1000 & 2455 & 1480 & 2610 & 735 \\
\hline
\end{tabular}

Notes: $-=$ not detected.

Table 3. CIPW norm of the ultramafic rocks from Leg 153.

\begin{tabular}{|c|c|c|c|c|c|c|c|c|c|c|c|}
\hline Sample no.: & 1 & 3 & 5 & 6 & 8 & 10 & 12 & 13 & 14 & 16 & Ultra \\
\hline C & 0.63 & - & 0.96 & 1.16 & - & - & 0.69 & - & 0.48 & - & 1.06 \\
\hline Or & 0.18 & 0.06 & 0.22 & 0.12 & 0.13 & 0.06 & 0.03 & 0.08 & 0.08 & 0.06 & 0.03 \\
\hline $\mathrm{Ab}$ & 0.87 & 0.55 & 0.59 & 0.59 & 0.48 & 0.87 & 0.69 & 0.77 & 0.69 & 0.76 & 0.24 \\
\hline An & 1.28 & 3.05 & 0.74 & 0.29 & 3.68 & 3.00 & 1.70 & 3.61 & 1.93 & 3.23 & 0.71 \\
\hline Opx & 27.31 & 17.71 & 24.48 & 21.37 & 19.82 & 16.52 & 26.59 & 21.33 & 19.75 & 18.31 & 28.73 \\
\hline $\mathrm{Cpx}$ & - & 0.66 & - & - & 1.22 & 1.31 & - & - & - & 2.61 & - \\
\hline Ol & 69.08 & 77.33 & 72.36 & 75.74 & 73.99 & 77.27 & 69.59 & 73.50 & 76.37 & 74.30 & 68.54 \\
\hline $\mathrm{Cm}$ & 0.58 & 0.59 & 0.58 & 0.66 & 0.63 & 0.91 & 0.66 & 0.63 & 0.63 & 0.68 & 0.63 \\
\hline II & 0.04 & 0.02 & 0.04 & 0.04 & 0.02 & 0.03 & 0.02 & 0.05 & 0.04 & 0.02 & 0.05 \\
\hline Ap & 0.03 & 0.03 & 0.03 & 0.03 & 0.03 & 0.03 & 0.03 & 0.03 & 0.03 & 0.03 & 0.01 \\
\hline TTI & 1.05 & 0.61 & 0.81 & 0.71 & 0.61 & 0.93 & 0.72 & 0.85 & 0.77 & 0.82 & 0.27 \\
\hline $\mathrm{An} *$ & 60 & 85 & 56 & 33 & 88 & 78 & 71 & 82 & 74 & 81 & 75 \\
\hline Mg\# & 90.9 & 90.5 & 90.2 & 90.5 & 90.1 & 90.3 & 89.9 & 90.5 & 90.5 & 90.4 & 89.8 \\
\hline $\mathrm{Ni} / \mathrm{Co}$ & 21.1 & 21.1 & 21.5 & 20.9 & 19.8 & 20.8 & 20.1 & 21.2 & 22.3 & 21.7 & 20.6 \\
\hline $\mathrm{Cr} / \mathrm{Ni}$ & 1.09 & 1.13 & 1,11 & 1.26 & 1.20 & 1.11 & 1.20 & 1.16 & 1.16 & 1.29 & 1.11 \\
\hline $\mathrm{Ti} / \mathrm{Zr}$ & 30 & 22 & 40 & 36 & 27 & 26 & 21 & 36 & 30 & 10 & 40 \\
\hline $\mathrm{Ti} / \mathrm{Cr}$ & 0.051 & 0.027 & 0.051 & 0.041 & 0.019 & 0.032 & 0.032 & 0.058 & 0.047 & 0.018 & 0.048 \\
\hline $\mathrm{V} / \mathrm{Cr}$ & 0.020 & 0.018 & 0.020 & 0.018 & 0.018 & 0.017 & 0.018 & 0.020 & 0.020 & 0.017 & 0.020 \\
\hline $\mathrm{Cu} / \mathrm{Zn}$ & 0.44 & 0.45 & 0.67 & 0.80 & 0.40 & 0.64 & 0.38 & 0.46 & 0.48 & 0.48 & 0.30 \\
\hline
\end{tabular}

Notes: $\mathrm{C}=$ corundum, $\mathrm{Or}=$ orthoclase, $\mathrm{Ab}=$ albite, $\mathrm{An}=$ anorthite, $\mathrm{Opx}=$ orthopyroxene, $\mathrm{Cpx}=$ clinopyroxene, $\mathrm{Ol}=$ olivine, $\mathrm{Il}=\mathrm{ilmenite}, \mathrm{Ap}=$ apatite, $\mathrm{An} *=$ normative anorthite content in plagioclase, $-=$ not determined, other abbreviations are defined in the text.

mafic rocks, with $\mathrm{Mg} \#=(\mathrm{Mg} /[\mathrm{Mg}+\mathrm{Fe}])$ between 89.8 and 90.9 . The Thornton-Tuttle Index (TTI) is generally below 1 (Table 3 ), and the dominant norm mineral of the $\mathrm{H}_{2} \mathrm{O}$-free calculated rocks is olivine (ca. 70-80 wt \%), followed by orthopyroxene (18-29 wt \%). Compared to the modal data (between 2 and $5 \mathrm{vol} \%$ ), normative clinopyroxene contents are generally too low. Plagioclase was not observed in any of the investigated thin sections. Actually, the relatively small amounts of $\mathrm{Na}, \mathrm{Ca}$, and $\mathrm{Al}$ in normative albite $(\mathrm{Ab})$ and anorthite
(An) are incorporated within the pyroxenes, including parts of normative corundum. Another part of $\mathrm{Al}$ is a constituent of the spinels.

Geochemical data from our samples suggest a moderate degree of depletion by partial melting, clearly lower than in more northern parts of the Mid-Atlantic Ridge, for instance, the Atlantic or Oceanographer Fracture Zones (Michael and Bonatti, 1985; Dick et al., 1984). The water-free, calculated average of all our analyses $(n=11)$ is nearly identical to the mean value of analyses from the less-refractory 
peridotite of Michael and Bonatti (1985) for the major-element oxides $\mathrm{SiO}_{2}, \mathrm{Fe}_{2} \mathrm{O}_{3}$, and $\mathrm{MgO}$. This indicates such an insufficient mobility of $\mathrm{Si}, \mathrm{Fe}$, and $\mathrm{Mg}$ that the bulk chemistry of the protolith was essentially not altered by partial melting and serpentinization. Some minor oxides, however, show lower concentrations in comparison with the Michael and Bonatti (1985), less-refractory peridotite: $\mathrm{Cr}_{2} \mathrm{O}_{3}$ is $86 \%, \mathrm{Al}_{2} \mathrm{O}_{3}$ is $62 \%, \mathrm{TiO}_{2}$ is $60 \%, \mathrm{CaO}$ is only $31 \%$, and $\mathrm{Na}_{2} \mathrm{O}$ is clearly higher, at $155 \%$. The striking differences among $\mathrm{Al}_{2} \mathrm{O}_{3}, \mathrm{CaO}$, and $\mathrm{Na}_{2} \mathrm{O}$ can be explained mainly by serpentinization; this fact is not surprising, because Michael and Bonatti (1985) considered the loss of some components through hydration in their model calculations.

As expected, the data from the three essential oxides $\left(\mathrm{MgO}, \mathrm{SiO}_{2}\right.$, and $\left.\mathrm{Fe}_{2} \mathrm{O}_{3}\right)$ show a very low relative variance $(\mathrm{V}): \mathrm{MgO}(\mathrm{V}=1.25 \%)$, $\mathrm{SiO}_{2}(\mathrm{~V}=1.5 \%)$, and $\mathrm{Fe}_{2} \mathrm{O}_{3}(\mathrm{~V}=2.95 \%)$. The subordinate component $\mathrm{Al}_{2} \mathrm{O}_{3}$ shows a $\mathrm{V}=6.3 \%$. The most variable oxide is $\mathrm{CaO}(\mathrm{V}=$ $75 \%)$, followed by $\mathrm{K}_{2} \mathrm{O}(\mathrm{V}=64 \%), \mathrm{TiO}_{2}(\mathrm{~V}=34 \%), \mathrm{Na}_{2} \mathrm{O}(\mathrm{V}=$ $23 \%)$, and $\mathrm{P}_{2} \mathrm{O}_{5}(\mathrm{~V}=21 \%)$. Of the trace elements, $\mathrm{Ni}, \mathrm{Co}, \mathrm{V}$, and $\mathrm{Cr}$ show low variability $(\mathrm{V}=2.5 \%-5.7 \%)$. The highest variance values are from $\mathrm{Cl}(\mathrm{V}=56 \%), \mathrm{B}(48 \%), \mathrm{S}(32 \%)$, As and $\mathrm{Sb}(\mathrm{V} \sim 80 \%)$, and to $\mathrm{Ba}, \mathrm{Cu}$, and $\mathrm{Sr}(\mathrm{V}=40 \%-65 \%)$. The latter elements were especially influenced by subseafloor metamorphism, which leads to their supply by serpentinization, as for $\mathrm{Ca}, \mathrm{K}$, and $\mathrm{Na}$ (which are supplied or lost, depending upon the governing temperature conditions, and the varying rock/water relations). Conversely, the concentrations of the compatible elements are very uniform in all of the samples throughout the profile, and even ratios such as $\mathrm{Ni} / \mathrm{Co}, \mathrm{Cr} / \mathrm{Ni}$ or $\mathrm{V} / \mathrm{Cr}$ are very similar (Table 2). However, there is no good correlation between loss on ignition (LOI) and $\mathrm{Cl}(\mathrm{r}=-0.333$, where $\mathrm{r}=$ correlation coefficient). $\mathrm{Cl}$ is most probably incorporated in serpentine minerals, whereas $\mathrm{Ca}$ was irregularly lost during alteration of clinopyroxene and orthopyroxene. The result of this Ca loss is reflected in the values of the CIPW norm, with only four clinopyroxene-bearing samples (Table 2).

In general, the incompatible-element contents are very low, and are strongly depleted relative to primitive mantle composition (Hofmann, 1988). The following percentages were calculated from averages of 11 ultramafic rocks (Hofmann, 1988): $\mathrm{Na}=21 \%, \mathrm{~K}=46 \%$, $\mathrm{Sr}=13 \%, \mathrm{Ba}=16 \%, \mathrm{Ti}=9 \%$, and $\mathrm{Zr}=38 \%$. For instance, an average of $2.4 \mathrm{ppm} \mathrm{Sr}$ is much lower than the $\mathrm{Sr}$ content of seawater. The depletion of the incompatible elements may result not only from hydration, but also, to a certain extent, from partial melting processes. Obviously, primary deviations in the MARK mantle peridotite composition relative to the worldwide mean composition cannot be ruled out.

\section{MINERAL CHEMISTRY}

\section{Olivine}

This study dealt only with the relict minerals of samples from Hole 920D. All thin sections investigated contained a comparably high percentage $(10-20 \mathrm{vol} \%)$ of relatively fresh olivine grains that can be divided into two grain-size groups: (1) elongated, "augen"like grains, 3-5 mm long, and (2) more or less rounded grains, with a diameter of 1-2 mm.

Both olivine groups are prekinematic or synkinematic. According to Cannat et al. (1992), the elongation is the result of a strong plastic strain with translation, kink banding, and granulation under asthenospheric conditions. Recrystallization of the small grains and their partial kink-band formation, however, occurred in a lower temperature environment.

Microprobe analyses of 83 olivines from both groups (Table 4) show no significant differences in their chemical composition. Forsterite $(\mathrm{Fo})$ varied only between $89.4 \%$ and $90.2 \%$ or, if $\mathrm{Ni}_{2}\left(\mathrm{SiO}_{4}\right)$ is included, between $89.8 \%$ and $90.6 \%$ (mean values are $89.8 \%$ and $90.2 \%$, respectively). $\mathrm{Mg} \#$ varied between 89.9 and 90.7 (average 90.26). $\mathrm{NiO}$ varied between $0.29 \mathrm{wt} \%$ and $0.49 \mathrm{wt} \%$ (average 0.39 $\mathrm{wt} \%)$. This variation is considerably higher than that of the $\mathrm{MgO}$ content, and was probably caused by internal displacement during tec- tonic deformation and/or a hydrothermal influence. Some olivine grain profiles show unsystematic patterns: the core can be Ni rich or $\mathrm{Ni}$ poor, the rim may show the opposite behavior, but irregular or directed distributions across the grain also occur. $\mathrm{FeO}, \mathrm{MgO}$, and $\mathrm{MnO}$,

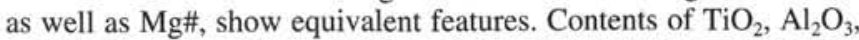
and $\mathrm{Cr}_{2} \mathrm{O}_{3}$ are very low, and the oxides are sometimes absent altogether. The $\mathrm{CaO}$ concentration varies between $0.01 \mathrm{wt} \%$ and 0.09 $\mathrm{wt} \%$. All of these features are characteristic of olivines from oceanic ultramafic rocks, according to Hamlyn and Bonatti (1980).

The mean values of our olivines (including $\mathrm{Mg \# )}$ are identical to those reported by Michael and Bonatti (1985) for olivines from lessrefractory peridotites from the North Atlantic part of the Mid-Atlantic Ridge, and, therefore, correspond well with the geochemical features of the whole rocks.

The more-refractory olivines from some more northern parts of the Mid-Atlantic Ridge contain higher Fo contents and, therefore, exhibit higher Mg\# values. The same is true for olivines from a more southern region (Cannat et al., 1992). The host rocks of these olivines are more-refractory peridotites than in the MARK region.

\section{Orthopyroxene}

Three main groups of orthopyroxene can be distinguished microscopically:

1. More or less rounded porphyroclasts (up to $10 \mathrm{~mm}$ long), with kink bands and clinopyroxene exsolution lamellae. Generally, the lamellae are partly, sometimes totally, transformed to serpentine. The outer rims are often replaced by talc and/or chrysotile.

2. Single grains (up to $2 \mathrm{~mm}$ diameter) within the matrix. The grains show undulatory extinction, kink banding, and contain very small clinopyroxene exsolutions.

3. Polygonal grain aggregates without deformation.

Orthopyroxene exsolution lamellae also occur in clinopyroxene.

A total of 74 prekinematic and postkinematic orthopyroxenes in thin sections from Hole $920 \mathrm{D}$ was investigated using the electron microprobe (Table 5). The data show only small differences in $\mathrm{Mg \#}$ (90.0-90.8), but they show a considerable variability of $\mathrm{Al}_{2} \mathrm{O}_{3}(3.5-$ $4.9 \mathrm{wt} \%), \mathrm{Cr}_{2} \mathrm{O}_{3}(0.55-1.15 \mathrm{wt} \%)$, and especially $\mathrm{CaO}(0.8-4.5 \mathrm{wt} \%)$ contents. $\mathrm{TiO}_{2}, \mathrm{MnO}$, and $\mathrm{NiO}$ contents also vary considerably.

No striking differences exist among the samples investigated, aside from the orthopyroxene in thin section 11 (Sample 920D-16R6, Piece 10), in which the contents of $\mathrm{Al}_{2} \mathrm{O}_{3}$ and $\mathrm{Cr}_{2} \mathrm{O}_{3}$ are clearly higher.

Calculation of the quadrilateral molecular pyroxene proportions according to the International Mineralogical Association proposal (Morimoto et al., 1989) shows in the more or less "normal" orthopyroxene $(\mathrm{n}=40 ; \mathrm{Mg} \#=90.2, \mathrm{Ca}=2.8$, where $\mathrm{Ca} \#=\mathrm{Ca} /[\mathrm{Ca}+\mathrm{Fe}+$ $\mathrm{Mg}]$ ):

Wollastonite $(\mathrm{Wo})=1.42-4.74 \mathrm{wt} \%$, mean $(\overline{\mathrm{x}})=2.83$, standard deviation $(\mathrm{s})= \pm 0.95, \mathrm{~V}= \pm 33.6 \%$

Enstatite $($ En) $=86.0-88.9 \mathrm{wt} \%, \overline{\mathrm{x}}=87.60, \mathrm{~s}= \pm 0.91, \mathrm{~V}=$ $\pm 1.04 \%$

Ferrosilite $(\mathrm{Fs})=8.9-11.3 \mathrm{wt} \%, \overline{\mathrm{x}}=9.57, \mathrm{~s}= \pm 0.37, \mathrm{~V}= \pm 3.87 \%$.

A few orthopyroxenes with higher Wo proportions $(\mathrm{n}=8 ; \mathrm{Mg} \#=$ 90.4, Ca\# = 6.8) show the following:

$$
\begin{aligned}
& \text { Wo }=5.1-8.8 w t \%, \bar{x}=6.81, s= \pm 1.44, V= \pm 21.1 \% \\
& E n=82.2-85.7 w t \%, \bar{x}=84.26, s= \pm 1.38, V= \pm 1.64 \% \\
& F s=8.7-9.2 w t \%, \bar{x}=8.93, s= \pm 0.166, V= \pm 1.86 \%
\end{aligned}
$$

In both cases, standard deviation and variance are very low for En and Fs, but Wo varies over wide ranges. The orthopyroxenes richer in Wo show an increase in their $\mathrm{Al}_{2} \mathrm{O}_{3}$ content. 
Table 4. Chemical composition of olivines from Hole 920D.

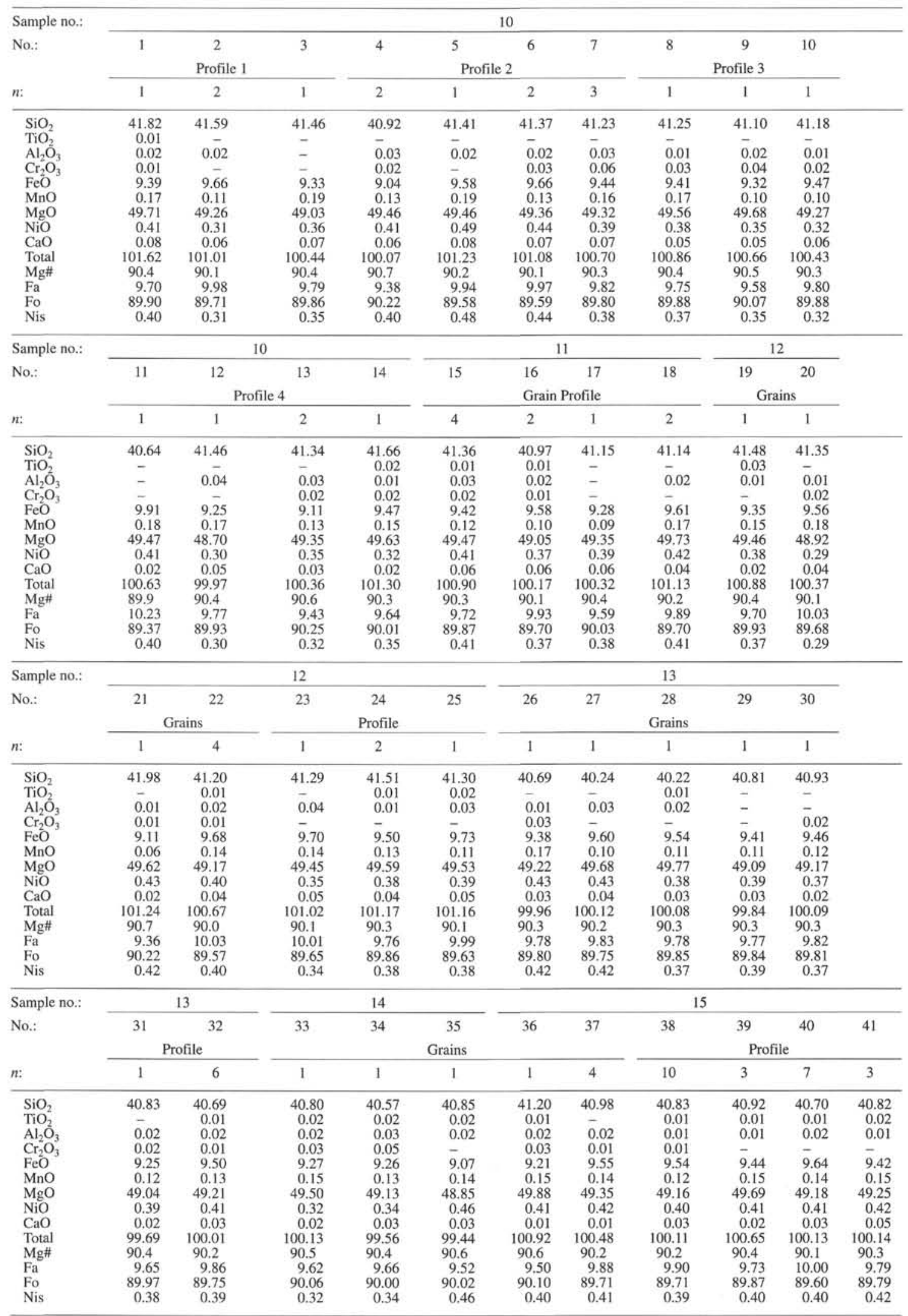

Notes: No, $=$ run number, $n=$ number of analyses performed, $\mathrm{Fo}=$ forsterite, $\mathrm{Fa}=$ fayalite, Nis $=\mathrm{Ni}$ silicate $\left(\mathrm{Ni}_{2}\left[\mathrm{SiO}_{4}\right]\right),-=$ not detected. 
Table 5. Chemical composition of orthopyroxenes, Hole 920D (prekinematic and porphyroclastic grains).

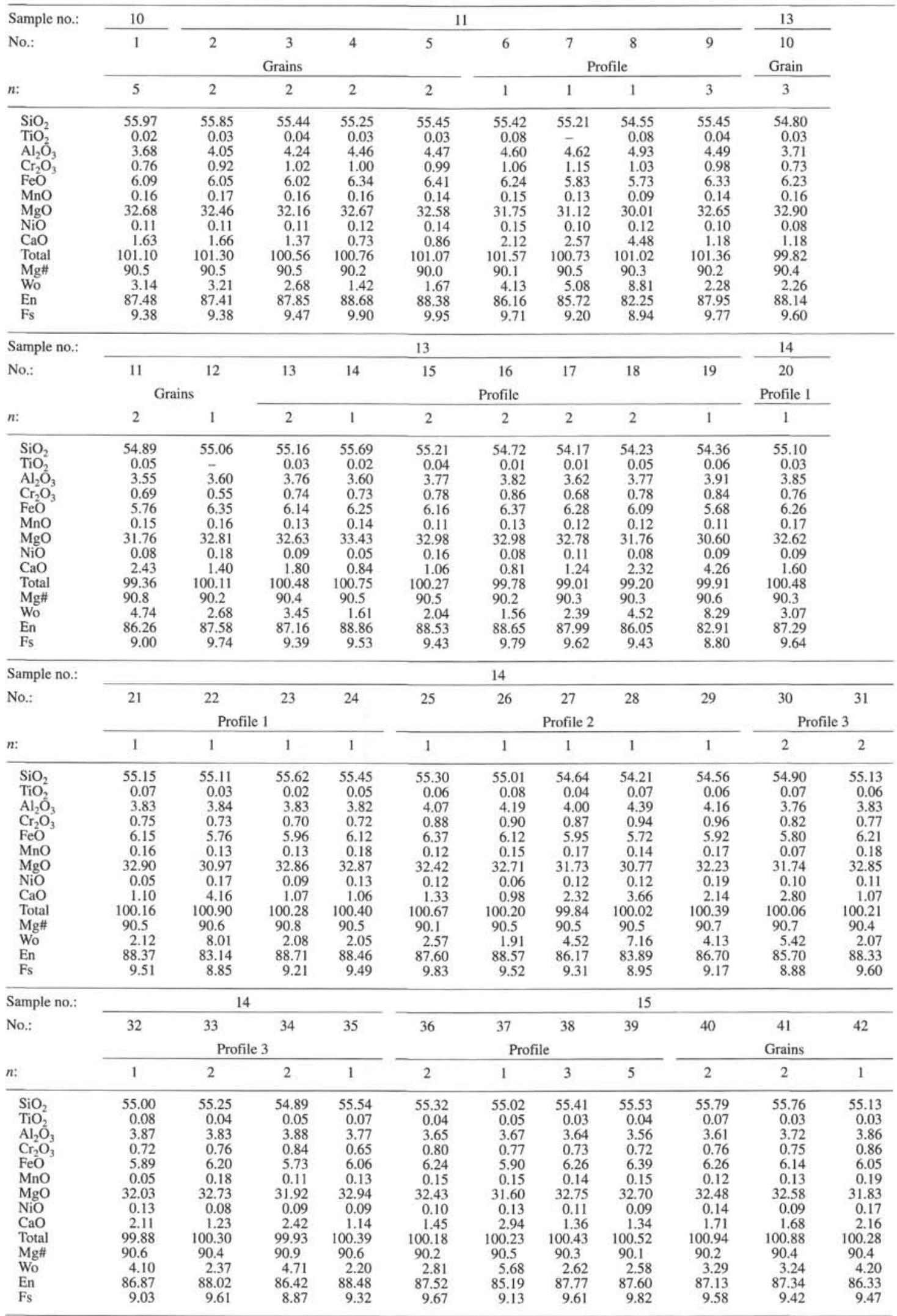

Note: Abbreviations are defined in the text and in Table 4. 
For both the orthopyroxene groups, no significant correlation between $\mathrm{CaO} / \mathrm{Cr}_{2} \mathrm{O}_{3}$ and $\mathrm{Al}_{2} \mathrm{O}_{3} / \mathrm{CaO}$ could be calculated. On the other hand, $\mathrm{Al}_{2} \mathrm{O}_{3}$ and $\mathrm{Cr}_{2} \mathrm{O}_{3}$ are very strongly correlated:

orthopyroxene $(\mathrm{n}=40): \mathrm{r}=+0.875 ;\left(3.5 \% \mathrm{Al}_{2} \mathrm{O}_{3}\right) /\left(0.69 \% \mathrm{Cr}_{2} \mathrm{O}_{3}\right)-$ $\left(5.0 \% \mathrm{Al}_{2} \mathrm{O}_{3}\right) /\left(1.14 \% \mathrm{Cr}_{2} \mathrm{O}_{3}\right)$

orthopyroxene $(\mathrm{n}=8): \mathrm{r}=+0.883 ;\left(3.5 \% \mathrm{Al}_{2} \mathrm{O}_{3}\right) /\left(0.72 \% \mathrm{Cr}_{2} \mathrm{O}_{3}\right)-$ $\left(5.0 \% \mathrm{Al}_{2} \mathrm{O}_{3}\right) /\left(1.12 \% \mathrm{Cr}_{2} \mathrm{O}_{3}\right)$.

High $\mathrm{CaO}$ and $\mathrm{Al}_{2} \mathrm{O}_{3} / \mathrm{Cr}_{2} \mathrm{O}_{3}$ contents in orthopyroxene can best be explained as the effects of high temperature in a dry system, only slightly influenced by pressure (Anastasiou and Seifert, 1972; Lindsley and Dixon, 1975; Obata, 1976; Sachtleben and Seck, 1981). Using the "Cr-Al-orthopyroxene" thermometer of Witt-Eickschen and Seck (1991), temperatures between $1245^{\circ}$ and $1340^{\circ} \mathrm{C}$, with an average of $1275^{\circ} \mathrm{C}$, were calculated for 8 selected orthopyroxenes. The Lindsley (1983) two-pyroxene thermometer, applied to 8 orthopyroxene/clinopyroxene pairs, resulted in a range from $1250^{\circ}$ to $1300^{\circ} \mathrm{C}$, and even the "classic" Kretz $(1963 ; 1982)$ two-pyroxene thermometer showed values between $1170^{\circ}$ and $1380^{\circ} \mathrm{C}$, with an average of $\sim 1300^{\circ} \mathrm{C}$. Only with the Wood and Banno (1973) method were higher temperatures $\left(1450^{\circ} \pm 25^{\circ} \mathrm{C}\right)$ calculated.

Five grain profiles clearly show a tendency in the $\mathrm{Fe}$ and $\mathrm{Mg}$ distribution toward higher contents in the marginal zone and lower contents in the core. The inverse is true for $\mathrm{Ni}$, to some degree. The behavior of $\mathrm{Ca}, \mathrm{Al}$, and $\mathrm{Cr}$ is irregular.

The microprobe results for a few polygonal grains and orthopyroxene exsolutions in clinopyroxene are listed in Table 6. The ortho-

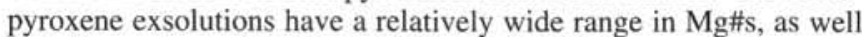
as in the contents of $\mathrm{Al}_{2} \mathrm{O}_{3}, \mathrm{FeO}, \mathrm{NiO}$, and $\mathrm{CaO}$. In contrast, however, the polygonal grains more or less correspond to the porphyroclastic orthopyroxenes, aside from their comparatively low $\mathrm{CaO}$ content.
A comparison with the data of Michael and Bonatti (1985) for orthopyroxenes from less-refractory and more-refractory peridotites from the northern section of the Mid-Atlantic Ridge shows a good correspondence between the mean value of all our orthopyroxenes and that from less-depleted peridotite; this is also consistent with the geochemical characteristics of MARK ultramafic rocks.

The orthopyroxene compositions of rocks from the $15^{\circ} 37^{\prime} \mathrm{N}$ region in the Mid-Atlantic Ridge axial valley (Cannat et al., 1992), which include strongly depleted peridotites, for instance, are clearly more refractory, with $\mathrm{Mg \#}=91.8$ compared to a mean of $\mathrm{Mg} \#=90.2$ in orthopyroxene from the Kane area.

Table 6. Chemical composition of orthopyroxenes, Hole 920D (postkinematic grains and exsolutions).

\begin{tabular}{|c|c|c|c|c|c|c|}
\hline \multirow{3}{*}{$\begin{array}{l}\text { Sample no: } \\
\text { No.: }\end{array}$} & \multicolumn{3}{|c|}{12} & \multicolumn{3}{|c|}{13} \\
\hline & 43 & 44 & 45 & 46 & 47 & 48 \\
\hline & \multicolumn{2}{|c|}{ Polygonal grains } & \multicolumn{4}{|c|}{ Exsolutions in clinopyroxene } \\
\hline$n:$ & 3 & 3 & 1 & 1 & 1 & 1 \\
\hline $\mathrm{SiO}_{2}$ & 56.06 & 56.34 & 53.53 & 54.69 & 54.62 & 54.99 \\
\hline $\mathrm{TiO}_{2}^{2}$ & 0.03 & 0.04 & 0.02 & 0.03 & 0.07 & 0.07 \\
\hline $\mathrm{Al}_{2} \mathrm{O}_{3}$ & 3.53 & 3.63 & 5.20 & 3.74 & 3.86 & 3.79 \\
\hline $\mathrm{Cr}_{2} \mathrm{O}_{3}$ & 0.72 & 0.73 & 1.07 & 0.78 & 0.81 & 0.83 \\
\hline $\mathrm{FeO}$ & 6.37 & 6.26 & 7.21 & 6.38 & 6.05 & 5.58 \\
\hline $\mathrm{MnO}$ & 0.13 & 0.14 & 0.16 & 0.18 & 0.11 & 0.16 \\
\hline $\mathrm{MgO}$ & 33.10 & 32.97 & 31.12 & 32.74 & 32.34 & 31.60 \\
\hline $\mathrm{NiO}$ & 0.04 & 0.17 & 0.10 & 0.13 & 0.07 & 0.05 \\
\hline $\mathrm{CaO}$ & 0.99 & 1.13 & 1.90 & 1.13 & 1.55 & 3.12 \\
\hline Total & 100.97 & 101.41 & 100.31 & 99.80 & 99.48 & 100.19 \\
\hline Mg\# & 90.2 & 90.4 & 88.5 & 90.1 & 90.5 & 91.0 \\
\hline Wo & 1.90 & 2.17 & 3.73 & 2.18 & 3.01 & 6.04 \\
\hline En & 88.36 & 88.24 & 84.98 & 87.95 & 87.63 & 85.26 \\
\hline Fs & 9.74 & 9.59 & 11.29 & 9.87 & 9.36 & 8.70 \\
\hline
\end{tabular}

Note: Abbreviations are defined in text and Table 4.

Table 7. Chemical composition of clinopyroxenes, Hole 920D (prekinematic and porphyroclastic grains).

\begin{tabular}{|c|c|c|c|c|c|c|c|c|c|c|}
\hline \multirow{2}{*}{$\begin{array}{l}\text { Sample no.: } \\
\text { No.: }\end{array}$} & \multicolumn{9}{|c|}{10} & \multirow{3}{*}{$\begin{array}{c}11 \\
10 \\
\text { Profile }\end{array}$} \\
\hline & 1 & 2 & 3 & 4 & 5 & 6 & 7 & 8 & 9 & \\
\hline & \multicolumn{3}{|c|}{ Grains } & \multicolumn{6}{|c|}{ Profile } & \\
\hline$n:$ & 4 & 2 & 1 & 3 & 2 & 2 & 3 & 2 & 1 & 2 \\
\hline $\mathrm{SiO}_{2}$ & 52.04 & 52.42 & 51.82 & 52.15 & 52.36 & 52.02 & 51.83 & 51.93 & 52.45 & 51.47 \\
\hline $\mathrm{TiO}_{2}^{2}$ & 0.07 & 0.13 & 0.11 & 0.08 & 0.07 & 0.09 & 0.10 & 0.12 & 0.11 & 0.19 \\
\hline $\mathrm{Al}_{2} \mathrm{O}_{3}$ & 4.67 & 4.45 & 4.59 & 4.64 & 4.61 & 4.75 & 4.91 & 4.74 & 4.85 & 5.75 \\
\hline $\mathrm{Cr}_{2} \mathrm{O}_{3}$ & 1.15 & 1.11 & 1.16 & 1.16 & 1.06 & 1.22 & 1.26 & 1.18 & 1.17 & 1.57 \\
\hline $\mathrm{FeO}$ & 2.93 & 2.60 & 2.60 & 3.30 & 3.29 & 2.71 & 2.61 & 3.04 & 3.53 & 2.20 \\
\hline $\mathrm{MnO}$ & 0.07 & 0.07 & 0.07 & 0.11 & 0.10 & 0.10 & 0.12 & 0.10 & 0.13 & 0.10 \\
\hline $\mathrm{MgO}$ & 17.30 & 16.54 & 16.48 & 18.46 & 18.24 & 16.65 & 16.25 & 17.28 & 18.63 & 15.80 \\
\hline $\mathrm{NiO}$ & 0.03 & 0.06 & 0.11 & 0.06 & 0.08 & 0.06 & 0.06 & 0.07 & 0.07 & 0.04 \\
\hline $\mathrm{CaO}$ & 22.47 & 23.66 & 23.91 & 20.68 & 20.67 & 23.43 & 24.07 & 22.11 & 20.88 & 24.31 \\
\hline $\mathrm{Na}_{2} \mathrm{O}$ & 0.08 & 0.06 & 0.06 & 0.07 & 0.05 & 0.06 & 0.08 & 0.07 & 0.04 & 0.11 \\
\hline Total & 100.81 & 101.10 & 100.91 & 100.71 & 100.53 & 101.09 & 101.29 & 100.64 & 101.86 & 101.54 \\
\hline $\mathrm{Mg \#}$ & 91.3 & 91.9 & 91.9 & 90.9 & 90.8 & 91.6 & 91.7 & 91.0 & 90.4 & 92.8 \\
\hline $\mathrm{Ca}^{*} \#$ & 48.3 & 50.7 & 51.0 & 44.6 & 44.9 & 50.3 & 51.6 & 47.9 & 44.6 & 52.5 \\
\hline Wo & 45.95 & 48.49 & 48.80 & 42.15 & 42.40 & 47.98 & 49.28 & 45.45 & 42.01 & 50.53 \\
\hline En & 49.26 & 47.24 & 46.94 & 52.42 & 52.17 & 47.53 & 46.36 & 49.51 & 52.25 & 45.74 \\
\hline Fs & 4.79 & 4.27 & 4.26 & 5.43 & 5.43 & 4.49 & 4.36 & 5.04 & 5.74 & 3.73 \\
\hline Sample no.: & \multicolumn{2}{|c|}{11} & \multicolumn{4}{|c|}{12} & \multicolumn{4}{|c|}{13} \\
\hline \multirow[t]{3}{*}{ No.: } & 11 & 12 & 13 & 14 & 15 & 16 & 17 & 18 & 19 & 20 \\
\hline & \multicolumn{2}{|c|}{ Profile } & \multicolumn{4}{|c|}{ Grains } & \multicolumn{4}{|c|}{ Grains } \\
\hline & 6 & 2 & 3 & 2 & 1 & 1 & 2 & 2 & 3 & 3 \\
\hline $\mathrm{SiO}_{2}$ & 51.31. & 50.94 & 51.33 & 51.82 & 52.27 & 52.21 & 50.65 & 50.33 & 50.61 & 51.34 \\
\hline $\mathrm{TiO}_{2}^{2}$ & 0.15 & 0.16 & 0.10 & 0.10 & 0.07 & 0.08 & 0.09 & 0.08 & 0.10 & 0.13 \\
\hline $\mathrm{Al}_{2} \mathrm{O}_{3}$ & 5.51 & 5.73 & 5.03 & 5.02 & 4.68 & 5.01 & 4.98 & 5.11 & 4.46 & 4.57 \\
\hline $\mathrm{Cr}_{2} \mathrm{O}_{3}$ & 1.49 & 1.69 & 1.33 & 1.29 & 1.23 & 1.14 & 1.26 & 1.36 & 1.17 & 1.13 \\
\hline $\mathrm{FeO}$ & 2.42 & 2.67 & 2.61 & 2.61 & 2.89 & 3.51 & 2.46 & 2.43 & 2.49 & 2.44 \\
\hline $\mathrm{MnO}$ & 0.10 & 0.08 & 0.13 & 0.08 & 0.12 & 0.10 & 0.10 & 0.10 & 0.09 & 0.08 \\
\hline $\mathrm{MgO}$ & 15.73 & 16.30 & 15.80 & 15.91 & 16.85 & 19.13 & 16.29 & 15.80 & 16.60 & 16.18 \\
\hline $\mathrm{NiO}$ & 0.07 & 0.04 & 0.11 & 0.05 & 0.06 & 0.02 & 0.06 & 0.12 & 0.07 & 0.05 \\
\hline $\mathrm{CaO}$ & 24.48 & 23.89 & 24.54 & 24.26 & 23.35 & 19.62 & 24.02 & 24.46 & 23.94 & 24.38 \\
\hline $\mathrm{Na}_{2} \mathrm{O}$ & 0.10 & 0.09 & 0.06 & 0.08 & 0.06 & 0.07 & 0.08 & 0.09 & 0.10 & 0.08 \\
\hline Total & 101.36 & 101.59 & 101.04 & 101.22 & 101.58 & 100.89 & 99.99 & 99.88 & 99.63 & 100.38 \\
\hline Mg\# & 92.0 & 91.6 & 91.8 & 91.6 & 91.2 & 90.7 & 92.2 & 92.1 & 92.2 & 92.2 \\
\hline $\mathrm{Ca}^{*} \#$ & 52.8 & 51.3 & 52.7 & 52.3 & 49.9 & 42.4 & 51.4 & 52.7 & 50.9 & 52.0 \\
\hline Wo & 50.60 & 49,01 & 50.35 & 50.00 & 47.48 & 39.99 & 49.30 & 50.43 & 48.76 & 49.86 \\
\hline En & 45.33 & 46.58 & 45.27 & 45.68 & 47.75 & 54.26 & 46.60 & 45.50 & 47.13 & 46.11 \\
\hline Fs & 4.07 & 4.41 & 4.38 & 4.32 & 4.77 & 5.75 & 4.10 & 4.07 & 4.11 & 4.03 \\
\hline
\end{tabular}




\section{Clinopyroxene}

Clinopyroxene is mainly developed as laminated porphyroclasts (up to 3-4 $\mathrm{mm}$ in size) with orthopyroxene exsolutions. Postkinematic recrystallization formed a smaller proportion of fresh and colorless grains, which are sometimes twinned, and occur subordinately in an intragranular position. They might represent products of a subsolidus crystallization process, but Nicolas et al. (1980) have discussed for that a formation by a limited local melting event. Exsolution lamellae in orthopyroxene constitute a third group of clinopyroxenes.

Microprobe data from 82 , mainly prekinematic or synkinematic, clinopyroxenes are listed in Table 7, and show a range in $\mathrm{Mg} \#$ from 90.0 to 92.8 . These values are clearly greater than those from orthopyroxene. The high $\mathrm{Al}_{2} \mathrm{O}_{3}$ contents vary from 3.75 to $5.75 \mathrm{wt} \%$, and are reflected by normative $\mathrm{Ts}$ (= tschermakite, $\mathrm{Mg}$ or Ca tschermakite) from $7.9 \%$ to $16.9 \%$. The main components, $\mathrm{CaO}$ (19.1-24.7 wt $\%$ ) and $\mathrm{MgO}$ (15.7-19.6 wt\%), show a considerable variability, as do $\mathrm{FeO}(2.25-3.85 \mathrm{wt} \%)$ and $\mathrm{Cr}_{2} \mathrm{O}_{3}(0.85-1.7 \mathrm{wt} \%)$. Contents of the minor-element oxides $\mathrm{TiO}_{2}, \mathrm{MnO}, \mathrm{NiO}$, and $\mathrm{Na}_{2} \mathrm{O}$ vary quite substantially. The clinopyroxenes of thin section 11 (Sample 920D-16R6 , Piece 10 ) are distinguished by above average $\mathrm{Al}_{2} \mathrm{O}_{3}$ and $\mathrm{Cr}_{2} \mathrm{O}_{3}$ contents, as in the corresponding orthopyroxene. All clinopyroxenes in the samples investigated can be described as tschermakitic (chromian) diopsides.

The quadrilateral molecular pyroxene proportions, according to the IMA proposal (Morimoto et al., 1989), show a main group of clinopyroxenes with higher, and a subgroup with clearly lower Wo contents:

Main group $(\mathrm{n}=33 ; \mathrm{Mg} \#=91.6)$ :
Wo $=46-50.6 \mathrm{wt} \%, \overline{\mathrm{x}}=49.03, \mathrm{~s}= \pm 1.35, \mathrm{~V}= \pm 2.75 \%$

$\mathrm{En}=45.3-49.4 \mathrm{wt} \%, \overline{\mathrm{x}}=46.71, \mathrm{~s}= \pm 1.22, \mathrm{~V}= \pm 2.61 \%$

Fs $=3.7-4.8 w t \%, \bar{x}=4.26, s= \pm 0.27, V= \pm 6.39 \%$.

Subgroup $(\mathrm{n}=12 ; \mathrm{Mg} \#=90.6)$ :

Wo $=38.6-45.5 \mathrm{wt} \%, \overline{\mathrm{x}}=42.62, \mathrm{~s}= \pm 2.45, \mathrm{~V}= \pm 5.76 \%$

$\mathrm{En}=49.5-55.1 \mathrm{wt} \%, \overline{\mathrm{x}}=52.01, \mathrm{~s}= \pm 2.07, \mathrm{~V}= \pm 3.98 \%$

Fs $=4.8-6.3 w t \%, \bar{x}=5.37, s= \pm 0.43, V= \pm 7.95 \%$.

Standard deviations and variance for Wo and En are somewhat lower than for Fs, and the subgroup generally shows a greater compositional range. The clinopyroxene grains of this group show a dismemberment along cleavage planes and some dissolution features at these interruption zones, with the formation of antigorite, tremolite/ actinolite, and, in some places, brown amphibole. This has led to a certain loss of $\mathrm{Ca}$ from the central parts of the grains, but the mean $\mathrm{Al}_{2} \mathrm{O}_{3}$ contents are nearly equal in both groups.

Peters (1968) has described a good correlation between $\mathrm{Ca} /(\mathrm{Ca}+$ $\mathrm{Mg}$ ) (defined as $\mathrm{Ca}^{*} \#$, in Tables 7,8) and the $\mathrm{Al}_{2} \mathrm{O}_{3}$ contents in clinopyroxene from Alpine serpentinites. The following correlations were calculated for the two clinopyroxene groups from Hole 920D:

clinopyroxene $(n=33): r=+0.481 ; \mathrm{Ca}^{*} \#$ ranges from 46 at 3.94 wt $\% \mathrm{Al}_{2} \mathrm{O}_{3}$ to 52 at $4.89 \mathrm{wt} \% \mathrm{Al}_{2} \mathrm{O}_{3}$,

clinopyroxene $(n=12): r=-0.204 ; \mathrm{Ca}^{*} \#$ ranges from 40 at 4.83 wt $\% \mathrm{Al}_{2} \mathrm{O}_{3}$ to 48 at 4.63 wt $\% \mathrm{Al}_{2} \mathrm{O}_{3}$.

This opposite behavior can be explained by the partial alteration of the clinopyroxene subgroup.

$\mathrm{Al}_{2} \mathrm{O}_{3}$ and $\mathrm{Cr}_{2} \mathrm{O}_{3}$ exhibit a positive correlation, but not as strong as that in the orthopyroxene system, and both groups differ noticeably:

Table 7 (continued).

\begin{tabular}{|c|c|c|c|c|c|c|c|c|c|c|}
\hline \multirow{2}{*}{$\begin{array}{l}\text { Sample no.: } \\
\text { No.: }\end{array}$} & \multicolumn{3}{|c|}{14} & \multicolumn{7}{|c|}{15} \\
\hline & 21 & 22 & 23 & 24 & 25 & 26 & 27 & 28 & 29 & 30 \\
\hline & \multicolumn{3}{|c|}{ Grains } & \multicolumn{3}{|c|}{ Profile } & \multicolumn{4}{|c|}{ Grains } \\
\hline$n:$ & 2 & 2 & 2 & 2 & 2 & 1 & 4 & 3 & 1 & 1 \\
\hline $\begin{array}{l}\mathrm{SiO}_{2} \\
\mathrm{TiO}_{2} \\
\mathrm{Al}_{2} \mathrm{O}_{3} \\
\mathrm{Cr}_{2} \mathrm{O}_{3} \\
\mathrm{FeO} \\
\mathrm{MnO} \\
\mathrm{MgO} \\
\mathrm{NiO} \\
\mathrm{CaO} \\
\mathrm{Na}_{2} \mathrm{O} \\
\mathrm{Total} \\
\mathrm{Mg \#} \mathrm{Ca}^{*} \# \\
\mathrm{Wo} \\
\mathrm{En} \\
\mathrm{Fs}\end{array}$ & $\begin{array}{r}51.00 \\
0.12 \\
4.92 \\
1.37 \\
2.42 \\
0.07 \\
16.02 \\
0.04 \\
24.12 \\
0.08 \\
100.16 \\
92.2 \\
52.0 \\
49.86 \\
46.12 \\
4.02\end{array}$ & $\begin{array}{r}51.51 \\
0.10 \\
3.77 \\
0.87 \\
2.74 \\
0.13 \\
16.92 \\
0.06 \\
23.53 \\
0.08 \\
99.69 \\
91.7 \\
50.0 \\
47.69 \\
47.78 \\
4.53\end{array}$ & $\begin{array}{r}51.43 \\
0.14 \\
4.75 \\
1.13 \\
2.54 \\
0.10 \\
17.27 \\
0.08 \\
22.57 \\
0.09 \\
100.10 \\
92.4 \\
48.4 \\
46.33 \\
49.43 \\
4.24\end{array}$ & $\begin{array}{r}51.63 \\
0.10 \\
4.85 \\
1.24 \\
2.61 \\
0.13 \\
15.93 \\
0.06 \\
24.50 \\
0.11 \\
101.16 \\
91.6 \\
52.5 \\
50.17 \\
45.46 \\
4.37\end{array}$ & $\begin{array}{r}50.98 \\
0.12 \\
4.48 \\
1.21 \\
2.39 \\
0.08 \\
15.92 \\
0.07 \\
24.72 \\
0.12 \\
100.09 \\
92.2 \\
52.7 \\
50.61 \\
45.44 \\
3.95\end{array}$ & $\begin{array}{r}50.29 \\
0.05 \\
4.27 \\
1.13 \\
2.71 \\
0.07 \\
16.78 \\
0.08 \\
23.98 \\
0.13 \\
99.49 \\
91.7 \\
50.7 \\
48.39 \\
47.23 \\
4.38\end{array}$ & $\begin{array}{c}51.42 \\
0.11 \\
4.67 \\
1.28 \\
2.46 \\
0.10 \\
15.94 \\
0.07 \\
24.69 \\
0.11 \\
100.85 \\
92.0 \\
52.7 \\
50.49 \\
45.43 \\
4.08\end{array}$ & $\begin{array}{c}51.89 \\
0.11 \\
4.71 \\
1.18 \\
2.39 \\
0.07 \\
16.05 \\
0.04 \\
24.58 \\
0.13 \\
101.15 \\
92.3 \\
52.4 \\
50.31 \\
45.75 \\
3.94\end{array}$ & $\begin{array}{r}52.27 \\
0.09 \\
4.82 \\
1.26 \\
2.46 \\
0.05 \\
16.68 \\
0.11 \\
23.50 \\
0.13 \\
101.37 \\
92.4 \\
50.3 \\
48.21 \\
47.77 \\
4.02\end{array}$ & $\begin{array}{r}52.45 \\
0.09 \\
4.12 \\
1.19 \\
2.67 \\
0.10 \\
17.42 \\
0.08 \\
22.80 \\
0.11 \\
101.03 \\
92.1 \\
48.5 \\
46.29 \\
49.31 \\
4.40\end{array}$ \\
\hline \multirow{3}{*}{$\begin{array}{l}\text { Sample no.: } \\
\text { No.: }\end{array}$} & \multicolumn{7}{|c|}{15} & & & \\
\hline & 31 & 32 & 33 & 34 & 35 & 36 & 37 & & & \\
\hline & & & & Grains & & & & & & \\
\hline$n:$ & 2 & 2 & 2 & 1 & 3 & 3 & & & & \\
\hline $\begin{array}{l}\mathrm{SiO}_{2} \\
\mathrm{TiO}_{2} \\
\mathrm{Al}_{2} \mathrm{O}_{3} \\
\mathrm{Cr}_{2} \mathrm{O}_{3} \\
\mathrm{FeO} \\
\mathrm{MnO} \\
\mathrm{MgO} \\
\mathrm{NiO} \\
\mathrm{CaO} \\
\mathrm{Na}_{2} \mathrm{O} \\
\mathrm{Total} \\
\mathrm{Mg \#} \mathrm{Ca}^{*} \# \\
\mathrm{Wo} \\
\mathrm{En} \\
\mathrm{Fs}\end{array}$ & $\begin{array}{r}52.19 \\
0.12 \\
5.18 \\
1.33 \\
3.85 \\
0.11 \\
19.53 \\
0.07 \\
19.08 \\
0.06 \\
101.52 \\
90.0 \\
41.2 \\
38.63 \\
55.10 \\
6.27\end{array}$ & $\begin{array}{c}51.30 \\
0.10 \\
5.20 \\
1.39 \\
2.99 \\
0.09 \\
17.34 \\
0.09 \\
22.17 \\
0.13 \\
100.80 \\
91.2 \\
47.9 \\
45.46 \\
49.60 \\
4.94\end{array}$ & $\begin{array}{r}51.41 \\
0.12 \\
5.10 \\
1.35 \\
2.48 \\
0.09 \\
15.82 \\
0.06 \\
24.35 \\
0.11 \\
100.89 \\
91.9 \\
52.5 \\
50.30 \\
45.55 \\
4.15\end{array}$ & $\begin{array}{c}50.99 \\
0.11 \\
5.16 \\
1.44 \\
2.79 \\
0.13 \\
15.92 \\
0.02 \\
24.38 \\
0.12 \\
101.06 \\
91.1 \\
52.4 \\
49.94 \\
45.40 \\
4.66\end{array}$ & $\begin{array}{r}51.81 \\
0.09 \\
4.44 \\
1.21 \\
3.01 \\
0.04 \\
17.77 \\
0.03 \\
22.48 \\
0.11 \\
100.99 \\
91.3 \\
47.6 \\
45.33 \\
49.87 \\
4.80\end{array}$ & $\begin{array}{r}51.39 \\
0.10 \\
5.02 \\
1.39 \\
2.70 \\
0.10 \\
16.26 \\
0.09 \\
24.10 \\
0.10 \\
101.25 \\
91.5 \\
51.6 \\
49.21 \\
46.32 \\
4.47\end{array}$ & $\begin{array}{r}51.67 \\
0.11 \\
4.65 \\
1.24 \\
2.60 \\
0.08 \\
16.28 \\
0.04 \\
24.19 \\
0.10 \\
100.96 \\
91.8 \\
51.7 \\
49.42 \\
46.31 \\
4.27\end{array}$ & & & \\
\hline
\end{tabular}

Note: Abbreviations are defined in Table 4 and in the text. 
Table 8. Chemical composition of clinopyroxenes, Hole 920D (postkinematic grains and exsolutions).

\begin{tabular}{|c|c|c|c|c|c|c|c|c|}
\hline \multirow{2}{*}{$\begin{array}{l}\text { Sample no.: } \\
\text { No.: }\end{array}$} & \multicolumn{2}{|c|}{14} & \multicolumn{5}{|c|}{15} & \multirow{2}{*}{$\begin{array}{l}13 \\
45\end{array}$} \\
\hline & 38 & 39 & 40 & 41 & 42 & 43 & 44 & \\
\hline & \multicolumn{3}{|c|}{ Exsolutions in orthopyroxene } & \multicolumn{5}{|c|}{ Postkinematic, intergranular } \\
\hline$n:$ & 1 & 1 & 1 & 1 & 1 & 3 & 2 & 1 \\
\hline $\mathrm{SiO}_{2}$ & 51.95 & 51.68 & 50.97 & 51.77 & 52.17 & 51.73 & 51.21 & 51.90 \\
\hline $\mathrm{TiO}_{2}^{2}$ & 0.13 & 0.12 & 0.13 & 0.09 & 0.08 & 0.12 & 0.07 & 0.01 \\
\hline $\mathrm{Al}_{2}^{2} \mathrm{O}_{3}$ & 3.91 & 4.49 & 5.11 & 4.41 & 4.78 & 4.43 & 4.23 & 4.39 \\
\hline $\mathrm{Cr}_{2} \mathrm{O}_{3}$ & 0.99 & 1.15 & 1.34 & 1.13 & 1.20 & 1.16 & 1.07 & 1.10 \\
\hline $\mathrm{FeO}$ & 2.25 & 2.48 & 2.70 & 3.33 & 3.25 & 2.88 & 3.00 & 3.45 \\
\hline $\mathrm{MnO}$ & 0.08 & 0.10 & 0.08 & 0.11 & 0.09 & 0.10 & 0.07 & 0.08 \\
\hline $\mathrm{MgO}$ & 16.69 & 16.39 & 16.13 & 18.90 & 18.13 & 17.31 & 17.58 & 19.65 \\
\hline $\mathrm{NiO}$ & 0.04 & 0.03 & 0.11 & 0.09 & 0.15 & 0.08 & 0.10 & - \\
\hline $\mathrm{CaO}$ & 24.29 & 23.97 & 23.83 & 20.56 & 21.78 & 23.02 & 22.25 & 19.65 \\
\hline $\mathrm{Na}_{2} \mathrm{O}$ & 0.08 & 0.05 & 0.14 & 0.08 & 0.08 & 0.10 & 0.09 & 0.08 \\
\hline Total & 100.41 & 100.46 & 100.54 & 100.47 & 101.71 & 100.93 & 99.67 & 100.31 \\
\hline $\mathrm{Mg} \#$ & 93.0 & 92.2 & 91.4 & 91.0 & 90.9 & 91.4 & 91.3 & 91.0 \\
\hline $\mathrm{Ca}^{*} \#$ & 51.1 & 51.3 & 51.5 & 43.9 & 46.3 & 48.8 & 47.6 & 41.8 \\
\hline Wo & 49.22 & 49.12 & 49.11 & 41.44 & 43.81 & 46.52 & 45.25 & 39.51 \\
\hline En & 47.10 & 46.76 & 46.42 & 53.13 & 50.95 & 48.78 & 49.87 & 54.95 \\
\hline Fs & 3.68 & 4.12 & 4.47 & 5.43 & 5.24 & 4.70 & 4.88 & 5.54 \\
\hline
\end{tabular}

Note: Abbreviations are defined in the text and in previous tables.

clinopyroxene $(\mathrm{n}=33): \mathrm{r}=+0.333 ;\left(3.5 \% \mathrm{Al}_{2} \mathrm{O}_{3}\right) /\left(0.83 \% \mathrm{Cr}_{2} \mathrm{O}_{3}\right)-$ $\left(5.5 \% \mathrm{Al}_{2} \mathrm{O}_{3}\right) /\left(1.50 \% \mathrm{Cr}_{2} \mathrm{O}_{3}\right)$,

clinopyroxene $(\mathrm{n}=12): \mathrm{r}=+0.768 ;\left(3.5 \% \mathrm{Al}_{2} \mathrm{O}_{3}\right) /\left(0.89 \% \mathrm{Cr}_{2} \mathrm{O}_{3}\right)-$ $\left(5.5 \% \mathrm{Al}_{2} \mathrm{O}_{3}\right) /\left(1.37 \% \mathrm{Cr}_{2} \mathrm{O}_{3}\right)$.

This mineral-chemical similarity could be the expression of compatible conditions during crystallization.

Three grain profiles demonstrate the opposite distribution of $\mathrm{MgO}$ and $\mathrm{CaO}$ : the rims are $\mathrm{MgO}$ rich and $\mathrm{CaO}$ poor, whereas the cores are $\mathrm{MgO}$ poor and $\mathrm{CaO}$ rich. $\mathrm{FeO}$ seems to follow the trend of $\mathrm{MgO}$, but $\mathrm{Al}_{2} \mathrm{O}_{3}, \mathrm{Cr}_{2} \mathrm{O}_{3}$, and $\mathrm{NiO}$ show an indistinct behavior. The marginal decrease of $\mathrm{CaO}$ may be caused by aqueous fluids at higher temperatures, and the subsequent loss of a Ca-rich solution.

The correspondence between the chemical composition of the Kane clinopyroxene and the data of Michael and Bonatti (1985) for less-depleted peridotites is not very satisfactory, but the $\mathrm{Mg} \#$ values lie close together (Kane: $\mathrm{Mg} \#=91.3$; Michael and Bonatti [1985]: $\mathrm{Mg} \#=90.8$ ). Clinopyroxene from more-refractory ultramafic rocks show Mg\# values between 92.5 and 94 (Michael and Bonatti, 1985; Cannat et al., 1992). This is not surprising, when one considers the sensitivity of the relatively complex clinopyroxene system during partial melting, subsolidus, and hydration processes.

Some of the postkinematic intragranular clinopyroxenes (Table 8) exhibit relatively high $\mathrm{Al}_{2} \mathrm{O}_{3}, \mathrm{Cr}_{2} \mathrm{O}_{3}$, and $\mathrm{FeO}$ contents, as well as a lower $\mathrm{TiO}_{2}$ content and nearly equal $\mathrm{Mg \# s}$, but low $\mathrm{Ca}$ *\# values. These features are consistent with the formation of the postkinematic clinopyroxenes in a lower temperature regime than that of the prekinematic clinopyroxenes.

Only three exsolution lamellae were investigated. They all have low $\mathrm{FeO}$ but high $\mathrm{CaO}$ contents (high $\mathrm{Ca}^{*} \#$ values), and are relatively rich in $\mathrm{TiO}_{2}$. The clinopyroxene exsolutions are enriched in $\mathrm{TiO}_{2}$, $\mathrm{Al}_{2} \mathrm{O}_{3}$, and $\mathrm{Cr}_{2} \mathrm{O}_{3}$, in contrast with their host mineral, orthopyroxene. The lamellae are also somewhat enriched in $\mathrm{MgO}$ relative to $\mathrm{FeO}$. Consequently, $\mathrm{Mg} \#$ is slightly higher than it is in orthopyroxene. We can therefore state that clinopyroxene exsolution leads to a partial impoverishment of $\mathrm{Ti}, \mathrm{Mg}, \mathrm{Cr}$, and $\mathrm{Al}$ in orthopyroxene. The composition of clinopyroxene exsolutions does not differ essentially from the composition of the prekinematic to synkinematic clinopyroxene, aside from some smaller $\mathrm{Cr}$ proportions.

\section{Chromian Spinel}

$\mathrm{Cr}$ spinel is an integral, but irregularly distributed, accessory mineral in ultramafic rocks. It occurs mostly as larger, lobate, but more or less fractured, grains in more or less altered olivines and orthopyroxenes. The grains always show some dissolution and commonly show a thin overgrowth of secondary magnetite, resulting from serpentinization. Results of a limited number of analyses from only three samples are compiled in Table 9, and the hypothetical mineral end members are calculated.

All grains investigated are picotites, with only small variances within individual thin sections. Spinels from sample 11 (Sample 920D-16R-6, Piece 10) are lower in $\mathrm{Cr}_{2} \mathrm{O}_{3}$ and somewhat higher in $\mathrm{Al}_{2} \mathrm{O}_{3}$ than those from sample 15 (Sample 920D-22R-2, Piece 1D). In thin section 11, the spinels show normative hercynite. Calculations for sample 15, however, result in magnesiochromite (MCm) and ferrochromite $(\mathrm{Cm})$, with few exceptions. These differences do not support the opinions of Christiansen (1985) and Nicolas et al. (1980) about a metamorphic origin of both types of $\mathrm{Cr}$ spinel. Generally, the $\mathrm{Cr}$ spinels investigated are primary peridotite minerals, but in thin section $15(\mathrm{Cr} \#>30$, where $\mathrm{Cr} \#=\mathrm{Cr} /[\mathrm{Cr}+\mathrm{Al}])$, a certain recrystallization at lower pressure should have modified the original composition, because the negative correlation between $\mathrm{Cr} \#$ in spinel and $\mathrm{Al}_{2} \mathrm{O}_{3}$ in orthopyroxene is disturbed here. However, the two samples are not sufficient for a substantial statement to be made.

The mean element ratios for the $\mathrm{Cr}$ spinel groups are as follows $\left(\mathrm{Fe}^{3+} \#=\mathrm{Fe}^{3+} /\left[\mathrm{Fe}^{3+}+\mathrm{Cr}+\mathrm{Al}\right]\right)$ :

$$
\begin{aligned}
& \text { sample } 11(\mathrm{n}=11): \mathrm{Cr} \#=28.3, \mathrm{Mg} \#=67.6, \mathrm{Fe}^{3+} \#=1.96, \\
& \text { sample } 15(\mathrm{n}=18): \mathrm{Cr} \#=30.6, \mathrm{Mg} \#=68.2, \mathrm{Fe}^{3+\#}=1.37 .
\end{aligned}
$$

A geothermometric estimation of orthopyroxene-spinel pairs after equation 6 of Sachtleben and Seck (1981) gave $\sim 1180^{\circ} \mathrm{C}$ for sample 11 , and $\sim 1075^{\circ} \mathrm{C}$ for sample 15 . Both temperatures are lower than those calculated using pyroxene thermometry, and may reflect a certain element exchange between the spinels and the surrounding silicate minerals during subsolidus deformation and recrystallization.

\section{OXYGEN-ISOTOPE INVESTIGATIONS FROM AN ULTRAMAFIC PROFILE, HOLE 920D: ALTERATION BY SEAWATER}

Oxygen-isotope investigations from oceanic environments were first performed on samples dredged from the ocean floor (for example, Muehlenbachs and Clayton, 1971, 1972a, 1972b; Wenner and Taylor, 1973), and later on samples from borehole material (Muehlenbachs, 1976, 1977, 1978, 1979). The main results are the following: (1) Unaltered mid-ocean ridge basalt (MORB) samples give $\delta^{18} \mathrm{O}$ $=5.7 \%_{0} \pm 0.3 \%$ o (i.e., $\delta^{18} \mathrm{O}=5.7 \% \circ \pm 0.2 \%$ from Muehlenbachs and Clayton, 1972a, 1972b; Kyser et al. 1982; Ito et al., 1987; for the bulk mantle $\delta^{18} \mathrm{O}=5.5 \%$, from Mattey et al., 1994). (2) Alteration pro- 
Table 9. Chemical composition of $\mathrm{Cr}$ spinels, Hole 920D.

\begin{tabular}{|c|c|c|c|c|c|c|c|c|c|c|}
\hline \multirow{2}{*}{$\begin{array}{l}\text { Sample no.: } \\
\text { No.: }\end{array}$} & \multicolumn{10}{|c|}{11} \\
\hline & 1 & 2 & 3 & 4 & 5 & 6 & 7 & 8 & 9 & 10 \\
\hline $\mathrm{SiO}_{2}$ & 0.04 & 0.02 & 0.04 & 0.02 & 0.05 & 0.06 & - & 0.03 & 0.05 & 0.05 \\
\hline $\mathrm{TiO}_{2}$ & - & 0.06 & 0.03 & 0.07 & 0.04 & 0.05 & 0.02 & 0.08 & 0.03 & 0.06 \\
\hline $\mathrm{Al}_{2} \mathrm{O}_{3}$ & 41.37 & 41.63 & 41.14 & 42.01 & 41.59 & 42.70 & 43.14 & 42.40 & 42.73 & 42.97 \\
\hline $\mathrm{Cr}_{2} \mathrm{O}_{3}$ & 24.87 & 24.48 & 25.10 & 25.08 & 25.36 & 24.12 & 24.83 & 24.15 & 24.96 & 25.44 \\
\hline $\mathrm{Fe}_{2} \mathrm{O}_{3}$ & 2.74 & 3.41 & 2.27 & 1.70 & 1.96 & 1.73 & 1.41 & 2.37 & 1.55 & 0.85 \\
\hline $\mathrm{FeO}$ & 13.90 & 13.64 & 13.45 & 13.71 & 13.72 & 13.18 & 13.18 & 12.74 & 13.23 & 13.56 \\
\hline $\mathrm{MnO}$ & 0.20 & 0.14 & 0.20 & 0.21 & 0.14 & 0.18 & 0.24 & 0.16 & 0.14 & 0.17 \\
\hline $\mathrm{MgO}$ & 15.53 & 15.84 & 15.65 & 15.64 & 15.70 & 15.98 & 16.33 & 16.24 & 16.18 & 15.97 \\
\hline $\mathrm{NiO}$ & 0.27 & 0.29 & 0.21 & 0.25 & 0.13 & 0.20 & 0.22 & 0.26 & 0.24 & 0.24 \\
\hline $\mathrm{CaO}$ & - & 0.01 & - & 0.02 & 0.01 & - & - & 0.04 & - & 0.02 \\
\hline Total & 98.91 & 99.49 & 98.07 & 98.69 & 98.70 & 98.20 & 99.37 & 98.47 & 99.10 & 99.32 \\
\hline $\mathrm{Cr} \#$ & 28.7 & 28.3 & 29.0 & 28.6 & 29.0 & 27.5 & 27.8 & 27.6 & 28.2 & 28.4 \\
\hline $\mathrm{Mg \#}$ & 66.6 & 67.4 & 67.5 & 67.0 & 67.1 & 68.4 & 68.8 & 69.4 & 68.6 & 67.7 \\
\hline $\mathrm{Fe}^{3+\#}$ & 2.92 & 3.61 & 2.44 & 1.82 & 2.09 & 1.84 & 1.48 & 2.52 & 1.64 & 0.89 \\
\hline $\mathrm{Cm}$ & 27.95 & 27.36 & 28.42 & 28.20 & 28.57 & 27.13 & 27.45 & 27.12 & 27.80 & 28.35 \\
\hline $\mathrm{MCm}$ & - & - & - & 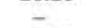 & - & - & - & - & - & - \\
\hline $\mathrm{Mt}$ & 2.93 & 3.62 & 2.44 & 1.82 & 2.10 & 1.84 & 1.48 & 2.53 & 1.64 & 0.90 \\
\hline $\mathrm{Hc}$ & 2.68 & 1.61 & 1.85 & 3.11 & 2.36 & 2.83 & 2.47 & 0.98 & 2.06 & 3.13 \\
\hline $\mathrm{Sp}$ & 66.44 & 67.41 & 67.29 & 66.87 & 66.97 & 68.20 & 68.60 & 69.37 & 68.50 & 67.62 \\
\hline Sample no.: & 11 & 12 & \multicolumn{8}{|c|}{15} \\
\hline No.: & 11 & 12 & 13 & 14 & 15 & 16 & 17 & 18 & 19 & 20 \\
\hline $\mathrm{SiO}_{2}$ & 0.04 & 0.04 & 0.01 & 0.07 & 0.03 & 0.07 & 0.08 & 0.06 & 0.05 & 0.03 \\
\hline $\mathrm{TiO}_{2}$ & 0.05 & 0.01 & 0.07 & 0.05 & 0.08 & 0.09 & 0.09 & 0.05 & 0.05 & 0.11 \\
\hline $\mathrm{Al}_{2} \mathrm{O}_{3}$ & 42.72 & 41.38 & 41.48 & 41.25 & 41.28 & 40.96 & 41.17 & 41.13 & 41.11 & 41.54 \\
\hline $\mathrm{Cr}_{2} \mathrm{O}_{3}$ & 24.78 & 25.93 & 26.90 & 27.23 & 26.85 & 27.48 & 26.93 & 27.13 & 26.95 & 26.75 \\
\hline $\mathrm{Fe}_{2} \mathrm{O}_{3}$ & 1.26 & 2.26 & 1.58 & 1.62 & 1.75 & 1.37 & 1.65 & 1.70 & 1.52 & 1.59 \\
\hline $\mathrm{FeO}$ & 13.26 & 12.23 & 13.57 & 13.32 & 13.01 & 13.13 & 12.91 & 12.72 & 13.15 & 12.84 \\
\hline $\mathrm{MnO}$ & 0.15 & 0.15 & 0.17 & 0.13 & 0.16 & 0.19 & 0.15 & 0.20 & 0.14 & 0.12 \\
\hline $\mathrm{MgO}$ & 16.04 & 16.67 & 16.03 & 16.15 & 16.19 & 16.07 & 16.14 & 16.28 & 16.15 & 16.34 \\
\hline $\mathrm{NiO}$ & 0.19 & 0.23 & 0.18 & 0.18 & 0.33 & 0.20 & 0.32 & 0.31 & 0.13 & 0.30 \\
\hline $\mathrm{CaO}$ & 0.02 & - & - & - & - & - & 0.02 & 0.07 & - & - \\
\hline Total & 98.50 & 98.90 & 100.01 & 100.00 & 99.68 & 99.55 & 99.44 & 99.66 & 99.25 & 99.61 \\
\hline $\mathrm{Cr} \#$ & 28.0 & 29.6 & 30.3 & 30.7 & 30.4 & 31.0 & 30.5 & 30.7 & 30.5 & 30.2 \\
\hline $\mathrm{Mg \#}$ & 68.3 & 70.8 & 67.8 & 68.4 & 68.9 & 68.6 & 69.0 & 69.5 & 68.6 & 69.4 \\
\hline $\mathrm{Fe}^{3+\#}$ & 1.34 & 2.40 & 1.66 & 1.70 & 1.85 & 1.45 & 1.74 & 1.80 & 1.62 & 1.68 \\
\hline $\mathrm{Cm}$ & 27.63 & 26.86 & 29.92 & 30.95 & 29.24 & 30.17 & 29.24 & 28.79 & 29.89 & 28.92 \\
\hline $\mathrm{MCm}$ & - & 2.11 & - & 0.34 & 0.73 & 0.64 & 0.98 & 1.58 & 0.30 & 0.92 \\
\hline Mt & 1.33 & 2.40 & 1.67 & 1.77 & 1.86 & 1.46 & 1.76 & 1.81 & 1.62 & 1.68 \\
\hline $\mathrm{Hc}$ & 3.20 & - & 0.76 & - & - & - & - & - & - & - \\
\hline $\mathrm{Sp}$ & 67.84 & 68.63 & 67.65 & 66.94 & 68.17 & 67.73 & 68.02 & 67.82 & 68.19 & 68.48 \\
\hline Sample no.: & \multicolumn{10}{|c|}{15} \\
\hline No.: & 21 & 22 & 23 & 24 & 25 & 26 & 27 & 28 & 29 & 30 \\
\hline $\mathrm{SiO}_{2}$ & 0.07 & 0.07 & 0.02 & 0.04 & 0.08 & 0.02 & 0.07 & 0.06 & 0.08 & 0.01 \\
\hline $\mathrm{TiO}_{2}$ & 0.04 & 0.09 & 0.07 & 0.08 & 0.09 & 0.06 & 0.09 & 0.06 & 0.07 & 0.08 \\
\hline $\mathrm{Al}_{2} \mathrm{O}_{3}$ & 40.74 & 41.21 & 41.22 & 41.04 & 41.37 & 41.03 & 41.20 & 41.09 & 41.63 & 40.99 \\
\hline $\mathrm{Cr}_{2} \mathrm{O}_{3}$ & 27.13 & 26.87 & 27.52 & 27.19 & 26.79 & 26.89 & 26.91 & 27.03 & 26.81 & 27.29 \\
\hline $\mathrm{Fe}_{2} \mathrm{O}_{3}$ & 1.67 & 2.02 & 1.24 & 1.06 & 1.70 & 1.69 & 1.63 & 1.51 & 1.37 & 1.11 \\
\hline $\mathrm{FeO}$ & 13.55 & 13.22 & 13.49 & 13.05 & 12.92 & 12.84 & 13.22 & 12.96 & 13.23 & 13.36 \\
\hline $\mathrm{MnO}$ & 0.17 & 0.17 & 0.16 & 0.20 & 0.12 & 0.14 & 0.11 & 0.16 & 0.14 & 0.16 \\
\hline $\mathrm{MgO}$ & 15.78 & 16.10 & 15.99 & 16.01 & 16.27 & 16.25 & 16.08 & 16.16 & 16.09 & 15.93 \\
\hline $\mathrm{NiO}$ & 0.22 & 0.25 & 0.27 & 0.23 & 0.19 & 0.31 & 0.20 & 0.24 & 0.21 & 0.22 \\
\hline $\mathrm{CaO}$ & 0.01 & 0.01 & 0.03 & 0.01 & 0.01 & - & - & - & 0.04 & - \\
\hline Total & 99.36 & 100.00 & 100.01 & 98.91 & 99.54 & 99.21 & 99.51 & 99.26 & 99.67 & 99.14 \\
\hline $\mathrm{Cr} \#$ & 30.9 & 30.4 & 30.9 & 30.8 & 30.3 & 30.5 & 30.5 & 30.6 & 30.2 & 30.9 \\
\hline $\mathrm{Mg \#}$ & 67.5 & 68.4 & 67.9 & 68.6 & 69.2 & 69.3 & 68.4 & 69.0 & 68.4 & 68.0 \\
\hline $\mathrm{Fe}^{3+\#}$ & 1.78 & 2.13 & 1.32 & 1.13 & 1.80 & 1.79 & 1.72 & 1.59 & 1.44 & 1.18 \\
\hline $\mathrm{Cm}$ & 30.50 & 29.49 & 30.67 & 30.35 & 29.06 & 28.93 & 29.86 & 29.52 & 30.00 & 30.64 \\
\hline $\mathrm{MCm}$ & - & 0.53 & - & 0.19 & 0.94 & 1.16 & 0.30 & 0.79 & - & - \\
\hline $\mathrm{Mt}$ & 1.78 & 2.15 & 1.32 & 1.13 & 1.82 & 1.80 & 1.74 & 1.60 & 1.45 & 1.18 \\
\hline $\mathrm{Hc}$ & 0.34 & - & 0.21 & - & - & - & - & - & 0.19 & 0.27 \\
\hline $\mathrm{Sp}$ & 67.38 & 67.83 & 67.80 & 68.33 & 68.18 & 68.11 & 68.10 & 68.09 & 68.36 & 67.91 \\
\hline
\end{tabular}

Note: Abbreviations are defined in the text and in previous tables.

cesses by seawater at low temperatures (lower than $100^{\circ} \mathrm{C}$ ) produces $\delta^{18} \mathrm{O}>5.7 \%$, partly a strong $\mathrm{O}^{18}$ enrichment that depends on temperature and degree of alteration (whole-rock $\delta^{18} \mathrm{O}$ up to more than $10 \%$, smectite up to more than 20\%o, Muehlenbachs and Clayton, 1972a). Basalts show a correlation between $\delta^{18} \mathrm{O}$ and water content. (3) Alteration processes by seawater at higher temperatures decrease the ${ }^{18} \mathrm{O}$ content. The latter is the case for seawater-formed serpentine minerals (Wenner and Taylor, 1971, 1973; Margaritz and Taylor, 1974; Ikin and Harmon, 1983; Tsen-Fu Yuei et al., 1990).

In this study, $\delta^{18} \mathrm{O}$ was determined from samples of a harzburgitic serpentinite profile from Hole 920D. To separate relict minerals from each profile segment, samples with the lowest degree of alteration were selected. Insofar as possible, olivine, orthopyroxene, and cli- nopyroxene were separated, in addition to serpentine and spinel/magnetite.

All analyses were made in a nickel-glass line using $\mathrm{ClF}_{3}$ to liberate oxygen, which was converted to $\mathrm{CO}_{2}$, and measured in a delta $\mathrm{E}$ mass spectrometer. The $\delta^{18} \mathrm{O}$ data are related to SMOW (standard mean ocean water), as National Bureau of Standards quartz no. 28 was used. All analyses were made in duplicate. The reproducibility was better than $0.2 \%$.

The $\delta^{18} \mathrm{O}$ values are shown in Table 10 . We note the following features:

1. The $\delta^{18} \mathrm{O}$-values of the whole-rock samples decrease from the bottom of the profile (4.6\%) to the top (3.0\%o). 
Table 10. Oxygen-isotope data $\left(\delta^{18} \mathrm{O}\right)$ from harzburgites, Hole $920 \mathrm{D}$

\begin{tabular}{|c|c|c|c|c|c|c|c|}
\hline $\begin{array}{l}\text { Core, section, } \\
\text { piece }\end{array}$ & $\begin{array}{l}\text { Depth } \\
\text { (mbsf) }\end{array}$ & $\begin{array}{l}\text { Whole } \\
\text { rock }\end{array}$ & Olivine & Orthopyroxene & Clinopyroxene & Serpentine & Magnetite \\
\hline $3 \mathrm{R}-1,6$ & 18.2 & 3.0 & & 5.2 & - & - & 2.9 \\
\hline $5 \mathrm{R}-1,4$ & 37.1 & 2.9 & & & - & 2.8 & 3.1 \\
\hline $14 \mathrm{R}-4,6$ & 119.4 & 4.3 & 5.9 & 6.1 & 5.9 & 4.7 & 3.8 \\
\hline $16 \mathrm{R}-1,5 \mathrm{D}$ & 134.4 & 3.8 & 6.1 & 6.1 & 6.1 & 4.2 & 3.0 \\
\hline $19 \mathrm{R}-1,5$ & 162.7 & 3.3 & & 6.0 & - & 3.2 & - \\
\hline $20 \mathrm{R}-5,4$ & 177.6 & 4.0 & 6.1 & 6.1 & 5.9 & 3.7 & 3.1 \\
\hline $21 \mathrm{R}-2,5 \mathrm{~B}$ & 183.9 & 3.8 & 5.6 & 5.8 & 5.9 & 3.8 & 3.1 \\
\hline $22 \mathrm{R}-4,1 \mathrm{~B}$ & 192.1 & 4.6 & 5.5 & 5.9 & 5.8 & 5.3 & 3.6 \\
\hline
\end{tabular}

Note: $-=$ mineral fraction is not present.

2. The deepest sample 16 (Sample 920D-22R-4, Piece 1B) has a $\delta^{18} \mathrm{O}$ distinctly smaller than the mean $\delta^{18} \mathrm{O}$ value of fresh MORB $(5.7 \%$ ). We conclude that all these whole-rock samples were altered by circulating ocean waters, and the lowered $\delta^{18} \mathrm{O}$ values indicate that these ocean waters were heated.

3. This tendency toward lower ${ }^{18} \mathrm{O}$ contents is not uniform: the third sample from the top (sample 8: Sample 920D014R-4, Piece 6$)$, has the second highest $\delta^{18} \mathrm{O}(4.3 \%)$, much higher than values from samples taken nearby. The third sample from the bottom (sample 13: Sample 920D-20R-5, Piece 4) has the next highest value, $4.0 \%$ (Fig. 1). Low ${ }^{18} \mathrm{O}$ contents (relative to those of their neighbors) are obtained from samples 14 (Sample 920D-21R-2, Piece 5B), 12 (Sample 920D-19R-1, Piece 5), and the top samples 6 (Sample 920D-5R-1, Piece 4), and 5 (Sample 920D-3R-1, Piece 6), which must indicate localities of rechanneled flow of ocean water (i.e., in fault zones). The mineral separates can be divided into a group of relict minerals including olivine, orthopyroxene, and clinopyroxene, and most of the spinel/magnetite, and another group of consisting of alteration products, mainly serpentine and traces of extremely fine-grained magnetite formed during the serpentinization.

4. The primary formed, relict minerals (olivine, orthopyroxene, clinopyroxene) almost all have similar $\delta^{18} \mathrm{O}$, which is similar to MORB (5.7\%o); they seem to be slightly enriched in ${ }^{18} \mathrm{O}$ in the middle part of the profile. At the top of the profile, the orthopyroxene (sample 5: Sample 920D-3R-1, Piece $6 ; \delta^{18} \mathrm{O}=$ $5.2 \%$ ) is distinctly lowered.

5. Of the secondary minerals, serpentine has a $\delta^{18} \mathrm{O}$ range from $5.3 \%$ to $2.8 \%$, which is in the lower part of the range for oceanic serpentines reported by Tsen-Fu Yui et al. (1990; 3.0\% to $12.4 \%$ ), and in the middle of the range reported by Wenner and Taylor $(1973 ;+0.8 \%$ o to $6.7 \%$ ).

Of all minerals investigated, serpentine $\delta^{18} \mathrm{O}$ is most similar to whole-rock $\delta^{18} \mathrm{O}$. The whole-rock $\delta^{18} \mathrm{O}$ and the serpentine $\delta^{18} \mathrm{O}$ are correlated (Fig. 1). The whole-rock peak of sample 8 (Table 1) is also conspicuous in the serpentine profile.

The spinel/magnetite $\delta^{18} \mathrm{O}$ changes by only $0.9 \%$, and also exhibits a striking peak at the level of sample 8. Furthermore, two groups of samples have a constant $\delta^{18} \mathrm{O}$ (within the measurement error of $0.2 \%$ ), one with $3.6 \%$ and $3.8 \%$, and the second from $2.9 \%$ to $3.1 \%$.

The difference (serpentine - spinel/magnetite) is very small and decreases from $+1.7 \%$ to $-0.3 \%$ (within the measurement error). Using the temperature calibration of Wenner and Taylor (1973) for ${ }^{18} \mathrm{O}$ in coexisting serpentine and magnetite, we get (also with the largest difference, $1.7 \%$ ) unreasonably high temperatures, effectively $\infty$. This means that our spinel/magnetite samples contain nearly no secondary magnetite, formed during serpentinization (in isotopeexchange equilibrium with serpentine).

The isotope pattern in Figure 2 shows an obvious trend between whole rock and serpentine, but a great difference between olivine, or-

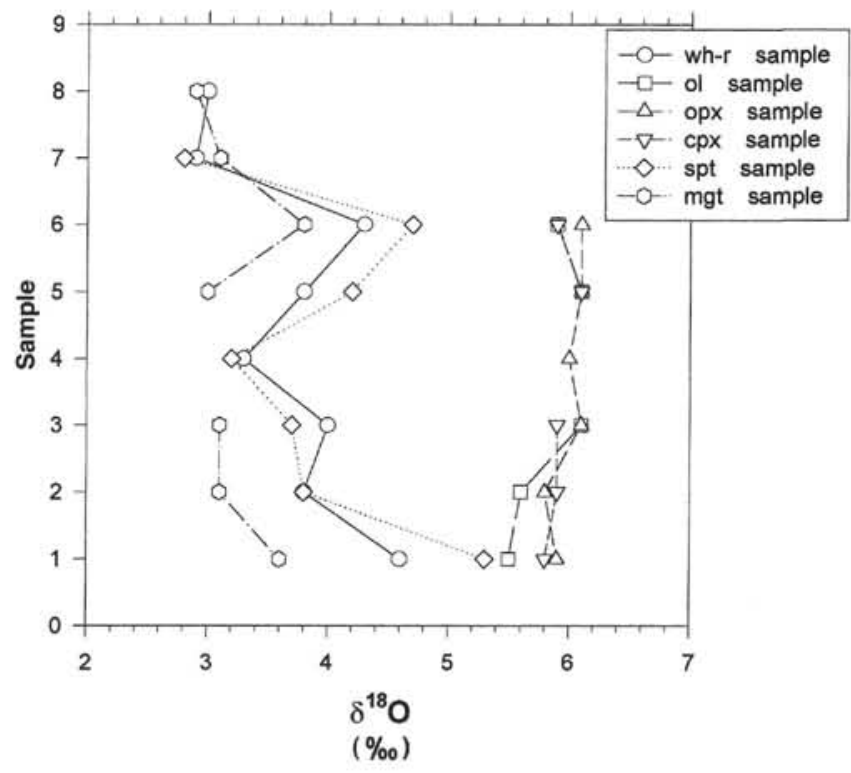

Figure $1 . \delta^{18} \mathrm{O}$ vs. harzburgite profile. Sample $1=$ Sample $153-920 \mathrm{D}-22 \mathrm{R}-4$ (Piece 1B), sample 2 = Sample 153-920D-21R-2 (Piece 5B), sample $3=$ Sample 153-920D-20R-5 (Piece 4), sample 4 = Sample 153-920D-19R-1 (Piece 5), sample 5 = Sample 153-920D-16R-1 (Piece 5D), sample 6 = Sample 153-920D-14R-4 (Piece 6), sample 7 = Sample 153-920D-5R-1 (Piece 4), and sample $8=$ Sample 153-920D-3R-1 (Piece 6). Abbreviations are defined as follows: wh- $\mathrm{r}=$ whole-rock, ol $=$ olivine, opx $=$ orthopyroxene, $\mathrm{cpx}=$ clinopyroxene, $\mathrm{spt}=$ serpentinite, $\mathrm{mgt}=$ magnetite .

thopyroxene, clinopyroxene, and spinel/magnetite, and the two groups of magnetite: (1) samples 8 and 16 , mean $\delta^{18} \mathrm{O}=3.7 \%$, which are the least altered whole-rock samples; (2) samples 14, 10, 6, and 5 , mean $\delta^{18} \mathrm{O}=3.0 \%$. Data from each group exhibit a surprisingly small degree of scatter.

If these alterations are due to the influence of circulating, hot, and possibly acidic ocean waters, then a relation between $\delta^{18} \mathrm{O}$ and some trace-element concentrations (especially of those trace elements that are more concentrated in the original ocean water) is possible. Figure 3 and Table 11 show a clearly negative correlation between wholerock $\delta^{18} \mathrm{O}$ and sulfur. In this regard, the results of sulfur-isotope analyses, which are in progress, may prove very exciting, because the ocean sulfate $\delta^{34} S$ of $20.3 \%$ is very different from the mantle value (near 0\%o).

The relations with other elements are not as convincing (Table 11): boron seems to be enriched with increasing alteration, similar to sulfur, and is characterized by greater scatter. Chlorine seems to be impoverished with increasing alteration, and strontium exhibits no significant correlation with alteration.

The water content (expressed as LOI) shows no correlation with the whole-rock $\delta^{18} \mathrm{O}$. Instead, we have two groups, containing exactly 


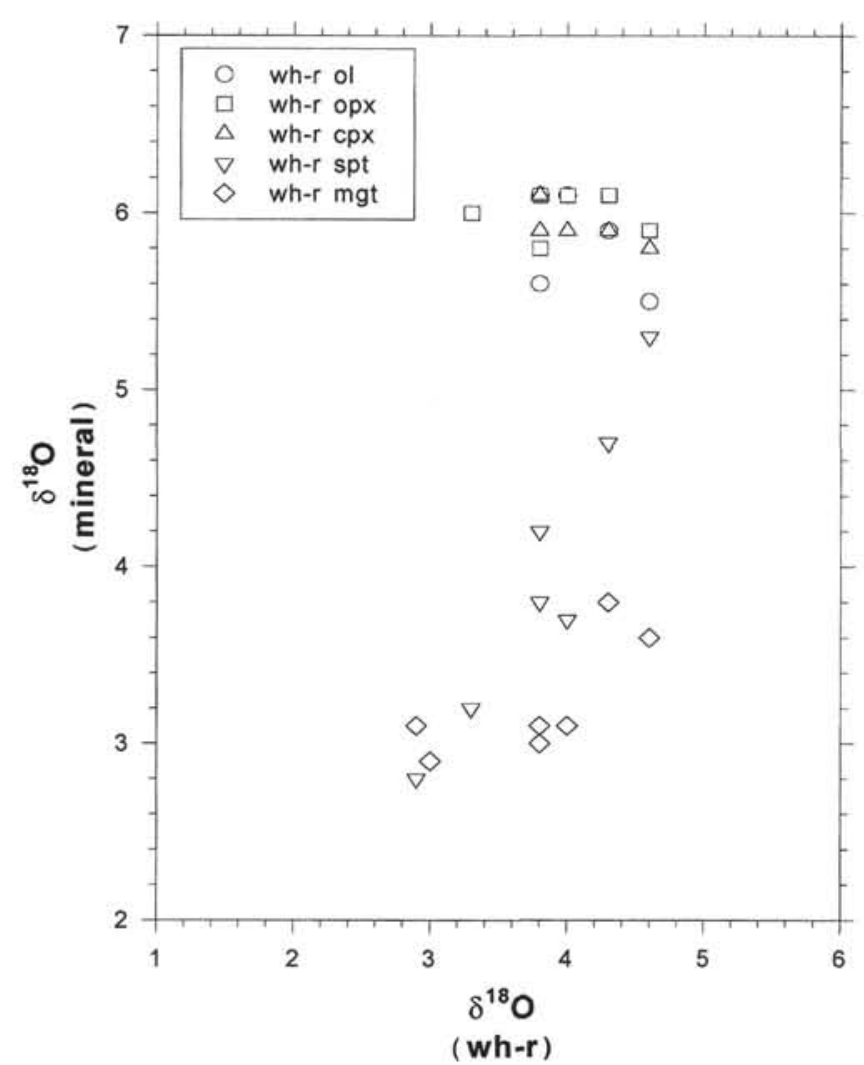

Figure 2. Whole-rock $\delta^{18} \mathrm{O}$ values plotted vs. $\delta^{18} \mathrm{O}$ values from different minerals. Alteration processes decreased whole-rock $\delta^{18} \mathrm{O}$ values. The relict minerals olivine, clinopyroxene, and orthopyroxene (in contrast to serpentinite and magnetite) underwent almost no oxygen alteration in the different levels of the profile. Abbreviations are defined in Figure 1.

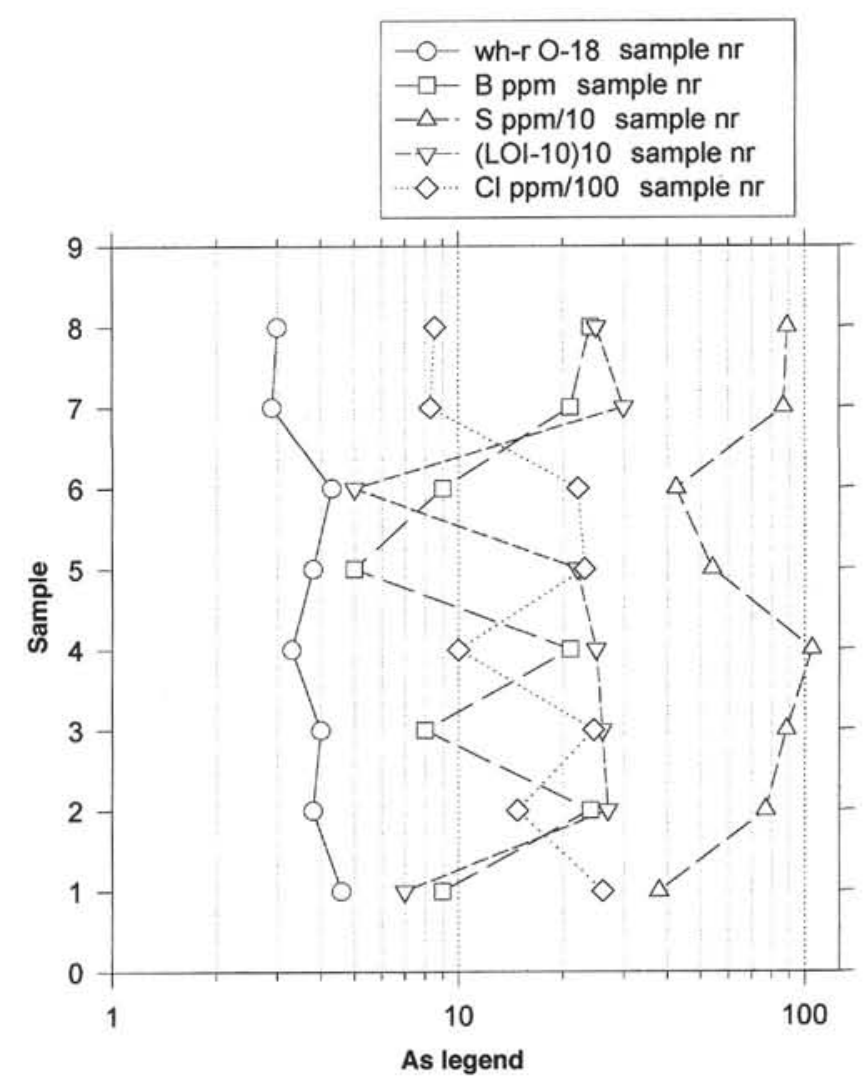

Figure 3. Whole-rock $\delta^{18} \mathrm{O}, \mathrm{B}, \mathrm{S} / 10, \mathrm{LOI}-10$, and $\mathrm{Cl} / 10$ plotted vs. the sample profile of harzburgites (see Fig. 1). The alteration seems to increase (in a different manner) with the contents of $\mathrm{B}, \mathrm{S}$, and $\mathrm{Cl}$, and with LOI. Abbreviations are defined in Figure 1.

Table 11. Whole-rock $\delta^{18} \mathrm{O}$ and the content of $\mathrm{B}, \mathrm{S} / 10, \mathrm{Cl} / 100, \mathrm{Sr}$, and $(\mathrm{LOI}-10) \cdot 10$ from harzburgites, Hole 920D.

\begin{tabular}{rcrrrrr}
\hline Core & $\begin{array}{c}\text { Whole-rock } \\
\delta^{18} \mathrm{O}\end{array}$ & $\mathrm{B}(\mathrm{ppm})$ & $\begin{array}{c}\mathrm{S} / 10 \\
(\mathrm{ppm})\end{array}$ & $(\mathrm{LOI}-10) \cdot 10$ & $\begin{array}{c}\mathrm{Cl} / 100 \\
(\mathrm{ppm})\end{array}$ & $\mathrm{Sr}$ \\
\hline 3R & 3.0 & 24 & 89.0 & 25 & 8.55 & 2.47 \\
5R & 2.9 & 21 & 87.0 & 30 & 8.30 & 0.93 \\
14R & 4.3 & 9 & 42.5 & 5 & 22.10 & 1.45 \\
16R & 3.8 & 5 & 54.0 & 22 & 23.10 & 1.21 \\
19R & 3.3 & 21 & 105.0 & 25 & 10.00 & 4.65 \\
20R & 4.0 & 8 & 88.5 & 26 & 24.55 & 2.28 \\
21R & 3.8 & 24 & 77.0 & 27 & 14.80 & 2.59 \\
22R & 4.6 & 9 & 38.0 & 7 & 26.10 & 2.47 \\
\hline
\end{tabular}

the same samples as the two groups of magnetite $\delta^{18} \mathrm{O}$ : high magnetite $\delta^{18} \mathrm{O}(3.7 \%)$ is associated with low LOI, and vice versa. The difference in the LOI values is small.

\section{Isotope Temperatures}

Assuming isotope-exchange equilibrium, we have calculated isotope temperatures for the mineral pairs clinopyroxene (diopside) and magnetite (spinel). We used the relation of Bottinga and Javoy (1975) for anhydrous minerals for temperatures (T) higher than $500^{\circ} \mathrm{C}$ and the coefficient for these minerals given by Matthews et al. (1983):

$$
\Delta^{18} \mathrm{O}(\text { clinopyroxene }- \text { magnetite })=4.03 \cdot 10^{6} / \mathrm{T}^{2}
$$

We get reasonable temperatures (Table 12), which cluster around $1100^{\circ}$ and $900^{\circ} \mathrm{C}$. However, one must consider that we do not have "pure" magnetite, but $\mathrm{Cr}$ spinel with a different composition, but the same crystal structure. Therefore, the calculation is affected by a partial uncertainty.

It is also difficult to obtain temperatures from the clinopyroxeneolivine pair. The olivine $\delta^{18} \mathrm{O}$ values (Table 10 ) form two groups, and the group around $6.0 \%$ does not differ from the $\delta^{18} \mathrm{O}$ of orthopyroxene and clinopyroxene. It is known that the $\delta^{18} \mathrm{O}$ values of clinopyroxene and orthopyroxene at such high temperatures do not differ (Bottinga and Javoy, 1975). The lack of a difference between pyroxene and olivine raises the question whether, at this level, these minerals were formed in isotope-exchange equilibrium. Examples of coexisting minerals of oceanic samples with clear disequilibrium have been reported (Muehlenbachs and Clayton, 1971).

The two deepest samples, samples 16 (Sample 920D-22R-4, Piece 1B), and 14 (Sample 920D-21R-2, Piece 5B), have lower $\delta^{18} \mathrm{O}$ in olivine, with a mean of $5.55 \%$; using the mean of orthopyroxene and clinopyroxene of $5.85 \%$, we get $\Delta^{18} \mathrm{O}=0.30 \%$. (If we tentatively 
Table 12. Calculated temperatures ( $T$ ) for clinopyroxene-magnetite, for the formation of the serpentines using $\delta^{18} \mathrm{O}=0$ and final $\delta^{18} \mathrm{O}$ of water equilibrated by formation of antigorite, Hole $920 \mathrm{D}$.

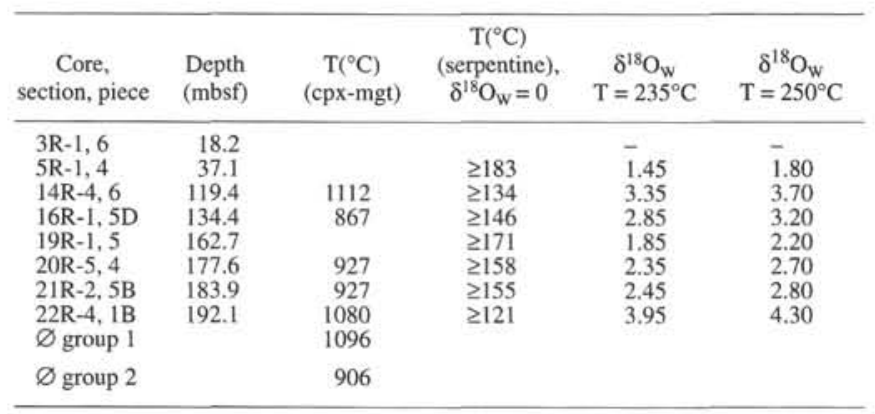

Note: $\mathrm{W}=$ water, $\mathrm{cpx}=$ clinopyroxene, $\mathrm{mgt}=$ magnetite. $\varnothing$ group $1=$ mean of Sample $14 \mathrm{R}-4$, (Piece 6) and 22R-4 (Piece IB); $\varnothing$ group $2=$ mean of Samples 16R-1 (Piece 5D), 20R-5 (Piece 4), and 21R-2 (Piece 5B).

calculate a temperature with the relation and coefficient $B=1.240$ of Bottinga and Javoy [1975], assuming an error of $0.2 \%$, we get a temperature of $1860^{\circ}$, with a variation between $-450^{\circ}$ and $+1200^{\circ}$ ).

To calculate the isotope temperature of serpentinization, we need a second mineral, with a $\delta^{18} \mathrm{O}$ value lower than that of serpentine, that formed in isotopic equilibrium with serpentine. Unfortunately, it was not possible to separate the very fine grained magnetite, which occurs only as traces and is intensively intergrown with serpentine. We tentatively calculated temperature, using the relation for serpentine-water from Wenner and Taylor $(1971,1973)$, and assuming $\delta^{18} \mathrm{O}=$ $0.0 \%$ for water as an extreme case (Table 12 ). We obtained temperatures between $121^{\circ}$ and $183^{\circ} \mathrm{C}$, increasing from bottom to top! Wenner and Taylor $(1971 ; 1973)$ estimated $125^{\circ} \mathrm{C}$ for lizardite and $180^{\circ} \mathrm{C}$ for chrysotile in Mid-Atlantic Ridge environments. Our samples, however, also contain antigorite (and some additional minerals, formed up to the transitional zone between greenschist and amphibolite facies). Wenner and Taylor $(1971 ; 1973)$ estimated $225^{\circ}$ and $235^{\circ} \mathrm{C}$ for antigorite. Pressure-temperature diagrams for the harzburgite system in equilibrium give a range from $250^{\circ}$ to $550^{\circ} \mathrm{C}$ for antigorite at a pressure of $3 \mathrm{kbar}$ (Bucher and Frei, 1994, p.153). We calculated the resulting $\delta^{18} \mathrm{O}$ for water that has attained isotopic equilibrium with the forming antigorite, assuming temperatures of $235^{\circ}$ and $250^{\circ} \mathrm{C}$ (Table 12), and obtained ranges from $1.45 \%$ to $3.95 \%$, and $1.80 \%$ o to $4.30 \%$. The seawater with $\delta^{18} \mathrm{O}=0 \%$ o had taken up ${ }^{18} \mathrm{O}$ by the formation of serpentine from olivine and other minerals.

\section{Water/Rock Ratios}

For the calculation of water/rock or better water/mineral (serpentine) ratios, we used the expressions of Taylor (1978). We assumed temperatures of $235^{\circ}, 250^{\circ}$, and $300^{\circ} \mathrm{C}$, and calculated ratios for closed and open systems in the sense of Taylor (Table 13). The final $\Delta^{18} \mathrm{O}$ (serpentine - water) is calculated for these temperatures using the equation of Wenner and Taylor (1973). For the deepest sample, only a small amount of water was available and active. The higher the sample position within the profile, the more water could penetrate to that point and cause isotope exchange (and, of course, mineral reorganization).

\section{CONCLUSIONS AND SUMMARY}

The ultramafic rock samples investigated are serpentinized harzburgites that contain $15 \%-30 \%$ relict minerals. Whole-rock geochemistry shows the normal pattern of less-depleted, refractory mantle peridotites. $\mathrm{Si}, \mathrm{Mg}$, and $\mathrm{Fe}$ contents are very uniform, as are the compatible trace elements, but contents of $\mathrm{K}, \mathrm{Na}, \mathrm{Ti}$, and especially $\mathrm{Ca}$, vary considerably. Partial anatexis of the primary mantle material and hydrothermal metamorphism of the depleted rocks lead to these irregularities, especially for $\mathrm{Ca}$.

The relict minerals olivine, orthopyroxene, and clinopyroxene show different morphological and genetic varieties, but their chemical compositions are relatively uniform within and between the individual samples, especially with respect to their $\mathrm{Mg} / \mathrm{Fe}$ ratios ( $\mathrm{Mg} \#)$ (Table 14).

Synkinematic or prekinematic olivine (with Fo $=89.8 \%-90.6 \%$ )

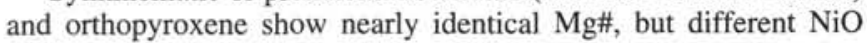
contents: in olivine, $\mathrm{NiO}=0.39 \mathrm{wt} \%(0.29-0.49 \mathrm{wt} \%)$, and in orthopyroxene, $\mathrm{NiO}=0.11 \mathrm{wt} \%(0.05-0.19 \mathrm{wt} \%)$. All these features are characteristic of less-refractory mantle minerals.

The contents of $\mathrm{Al}_{2} \mathrm{O}_{3}$ and $\mathrm{Cr}_{2} \mathrm{O}_{3}$ in orthopyroxene are strongly correlated $(\mathrm{r}=+0.88)$. CaO locally shows strikingly high concentrations (up to $4.5 \mathrm{wt} \%$ ), leading to a subgroup with Wo contents between 5 and $8.8 \mathrm{wt} \%$. Exsolution of clinopyroxene leads to a selective impoverishment of $\mathrm{Ti}, \mathrm{Mg}, \mathrm{Cr}$, and $\mathrm{Al}$ in orthopyroxene.

The porphyroclastic clinopyroxene can be classified as tschermakitic diopside because of its high $\mathrm{Al}_{2} \mathrm{O}_{3}$ content (3.75-5.75 wt\%). $\mathrm{Al}_{2} \mathrm{O}_{3}$ is well correlated with $\mathrm{Cr}_{2} \mathrm{O}_{3}$ in two groups $\left(\mathrm{r}_{1}=+0.33 ; \mathrm{r}_{2}=\right.$ +0.77 ), but not as strongly as in orthopyroxene. The general coincidence of these features with the equivalent data for orthopyroxene seems to reflect comparable conditions during the crystallization of both the pyroxenes. The behavior of $\mathrm{Mg}$ and $\mathrm{Ca}$ is clearly the opposite, and the scatter of $\mathrm{Ca}^{*}$ s is remarkable. Microprobe profiles through clinopyroxene grains show $\mathrm{MgO}$-rich and $\mathrm{CaO}$-poor rims, as well as $\mathrm{MgO}$-poor and $\mathrm{CaO}$-rich cores. The marginal decrease in $\mathrm{CaO}$ may be caused by reaction with fluids at higher temperatures, and probably also by partial melting.

In addition to the main group of clinopyroxenes $(\mathrm{Wo}=49, \mathrm{En}=$ $46.7, \mathrm{Fs}=4.3$ ), a subgroup exists that is clearly poorer in $\mathrm{Ca}$ and richer in $\mathrm{Mg}(\mathrm{Wo}=2.6, \mathrm{En}=52, \mathrm{Fs}=5.4)$. Both groups differ strongly in the correlation between $\mathrm{Ca} * \#$ and $\mathrm{Al}_{2} \mathrm{O}_{3}$ : the Wo-rich group has $\mathrm{r}$ $=+0.48$, and the Wo-poor group has $\mathrm{r}=-0.20$. This opposite behavior should be the result of partial alteration with a loss of $\mathrm{Ca}$.

The chromian-bearing spinels are typical picotites, but with certain differences. Hercynite-bearing samples are slightly poorer in $\mathrm{Cr}_{2} \mathrm{O}_{3}$, grains with somewhat higher $\mathrm{Cr}_{2} \mathrm{O}_{3}$ contents show some $\mathrm{Mg}$ chromite by calculation. All $\mathrm{Cr}$ spinels investigated must be considered as primary constituents of the peridotite, but they are altered to some degree.

Different temperatures were calculated using several geothermometric methods:

$1245^{\circ}-1340^{\circ} \mathrm{C}$, mean value $=1275^{\circ} \mathrm{C}$ ("Cr-Al-orthopyroxene" geothermometer; Witt-Eickschen and Seck, 1991),

$1250^{\circ}-1300^{\circ} \mathrm{C}$ (two-pyroxene geothermometer; Lindsley, 1983),

$1170^{\circ}-1380^{\circ} \mathrm{C}$, mean value $\approx 1300^{\circ} \mathrm{C}$ (two-pyroxene geothermometer; Kretz, 1963, 1982),

$1425^{\circ} \pm 25^{\circ} \mathrm{C}$ (Wood and Banno, 1973).

Aside from the Wood and Banno (1973) data, the temperature $\sim 1300^{\circ} \mathrm{C}$ may reflect the primary formation of prekinematic to synkinematic orthopyroxene and clinopyroxene in the protolith at pressures not greater than $10 \mathrm{kbar}$ (Anastasiou and Seifert, 1972; Wyllie, 1981).

In the orthopyroxene-chromian spinel system, applying the method of Sachtleben and Seck (1981) on the basis of $\mathrm{X}_{\mathrm{Mg}}, \mathrm{X}_{\mathrm{Al}}$, and $\mathrm{X}_{\mathrm{Cr}}$, the two $\mathrm{Cr}$-spinel-bearing samples yield temperatures of $1180^{\circ}$ and $1075^{\circ} \mathrm{C}$, respectively. With a certain restriction, the temperature estimate for the clinopyroxene-spinel/magnetite pair, using ${ }^{18} \mathrm{O}$ exchange, gave $1100^{\circ}$ and $900^{\circ} \mathrm{C}$. All these values may reflect subsolidus deformation and recrystallization processes, possibly in more than a single step. 
Table 13. Calculated water/rock ratios for serpentine for closed and open systems for different temperatures, Hole 920D.

\begin{tabular}{lrcccccc}
\hline $\begin{array}{c}\text { Core, } \\
\text { section, piece }\end{array}$ & $\begin{array}{c}\text { Depth } \\
(\mathrm{mbsf})\end{array}$ & $\begin{array}{c}(\mathrm{w} / \mathrm{r})_{\mathrm{cs}} \\
\mathrm{T}=235^{\circ} \mathrm{C}\end{array}$ & $\begin{array}{c}(\mathrm{w} / \mathrm{r})_{\mathrm{os}} \\
\mathrm{T}=235^{\circ} \mathrm{C}\end{array}$ & $\begin{array}{c}(\mathrm{w} / \mathrm{r})_{\mathrm{cs}} \\
\mathrm{T}=250^{\circ} \mathrm{C}\end{array}$ & $\begin{array}{c}(\mathrm{w} / \mathrm{r})_{\mathrm{cs}} \\
\mathrm{T}=250^{\circ} \mathrm{C}\end{array}$ & $\begin{array}{c}(\mathrm{w} / \mathrm{r})_{\mathrm{cs}} \\
\mathrm{T}=300^{\circ} \mathrm{C}\end{array}$ & $\begin{array}{c}(\mathrm{w} / \mathrm{r})_{\mathrm{os}} \\
\mathrm{T}=300^{\circ} \mathrm{C}\end{array}$ \\
\hline 3R-1,6 & 18.2 & - & - & - & - & - & - \\
$5 \mathrm{R}-1,4$ & 37.1 & $(3.67)$ & $(1.96)$ & $(2.95)$ & $(1.72)$ & $(1.93)$ & $(1.29)$ \\
$14 \mathrm{R}-4,6$ & 119.4 & 0.61 & 0.53 & 0.56 & 0.48 & 0.44 & 0.39 \\
16R-1,5D & 134.4 & 1.14 & 0.88 & 1.02 & 0.80 & 0.79 & 0.65 \\
19R-1,5 & 162.7 & $(2.69)$ & $(1.62)$ & $(2.26)$ & $(1.44)$ & $(1.58)$ & 1.12 \\
20R-5, 4 & 177.6 & 1.75 & 1.21 & 1.52 & 1.09 & 1.13 & 0.87 \\
21R-2,5B & 183.9 & 1.26 & 0.94 & 1.10 & 0.85 & 0.82 & 0.67 \\
22R-4, 1B & 192.1 & 0.09 & 0.08 & 0.08 & 0.08 & 0.07 & 0.06 \\
\hline
\end{tabular}

Notes: Numbers in parentheses represent values from serpentine with low $\delta^{18} \mathrm{O},-=$ not calculable, w/r $=$ water/rock ratio, $\mathrm{cs}=\mathrm{closed}, \mathrm{os}=$ open.

Table 14. Variation of mineral chemistry in the profile of Hole $920 \mathrm{D}$.

\begin{tabular}{|c|c|c|c|c|c|c|c|c|c|}
\hline \multirow[b]{2}{*}{ Sample no. } & \multirow[b]{2}{*}{$\begin{array}{c}\text { Core, } \\
\text { section, piece }\end{array}$} & \multirow[b]{2}{*}{$\begin{array}{l}\text { Depth } \\
\text { (mbsf) }\end{array}$} & \multicolumn{2}{|c|}{ Olivine } & \multicolumn{2}{|c|}{ Orthopyroxene } & \multicolumn{3}{|c|}{ Clinopyroxene } \\
\hline & & & $n$ & Mg\# & $n$ & Mg\# & $n$ & $\mathrm{Mg \#}$ & $\mathrm{Ca} \#$ \\
\hline 10 & $16 \mathrm{R}-1,5 \mathrm{D}$ & 134.4 & 20 & 90.33 & 5 & 90.53 & 20 & 91.29 & 48.26 \\
\hline 11 & $16 \mathrm{R}-6,6$ & 140.3 & 9 & 90.27 & 14 & 90.28 & 10 & 92.08 & 52.47 \\
\hline 12 & $19 \mathrm{R}-1,5$ & 162.7 & 11 & 90.19 & - & - & 7 & 91.34 & 50.75 \\
\hline 13 & $20 \mathrm{R}-5,4$ & 177.6 & 12 & 90.29 & 18 & 90.42 & 10 & 92.18 & 51.87 \\
\hline 14 & $21 R-2,5 B$ & 183.9 & 6 & 90.35 & 20 & 90.53 & 6 & 92.06 & 50.13 \\
\hline 15 & $22 \mathrm{R}-2, \mathrm{ID}$ & 192.1 & 28 & 90.21 & 16 & 90.26 & 29 & 91.60 & 50.82 \\
\hline
\end{tabular}

Note: Symbols and abbreviations are defined in previous tables.

The $\delta^{18} \mathrm{O}$ values are very uniform in the relict minerals olivine, orthopyroxene, and clinopyroxene, and values are similar to those of MORB, although slightly higher than for the bulk mantle. Wholerock samples, serpentine, and magnetite show clearly lower $\delta^{18} \mathrm{O}$ values, resulting from hydrothermal alteration at relatively high temperatures. The temperature determination of the serpentinization is restricted because only serpentine is available. Using some calculations and estimations about the water/rock ratio, a temperature range of $\pm 250^{\circ} \mathrm{C}$ could be probable.

\section{ACKNOWLEDGMENTS}

We are grateful to the Deutsche Forschungsgemeinschaft, Bonn, for considerable financial support of this work. H.-J. Bernhardt (Bochum) has greatly supported the microprobe investigations, B. Knipping (Clausthal), and G. Bombach, E. Rüdiger, and K. Volkmann (Freiberg) were responsible for the analytical procedure. C.-D.W. is grateful to all these colleagues and their institutions. The nickel-glass line was constructed, and the samples were separated and prepared by W. Tikhomirov; the mass spectrometric analyses were performed by R. Liebscher. For critical reviews and constructive comments, we thank E. Bonatti, J. Karson, and especially P. Kelemen and B. Wathen.

\section{REFERENCES}

Anastasiou, P., and Seifert, F., 1972. Solid solubility of $\mathrm{Al}_{2} \mathrm{O}_{3}$ in enstatite at high temperatures and $1-5 \mathrm{~kb}$ pressure. Contrib. Mineral. Petrol,, $34: 272-287$.

Aumento, F., and Loubat, H., 1971. The Mid-Atlantic Ridge near $45^{\circ}$ N. XVI. Serpentinized ultramafic intrusions. Can. J. Earth Sci., 8:631-663.

Bottinga, Y., and Javoy, M., 1975. Oxygen isotope partitioning among the minerals in igneous and metamorphic rocks. Rev. Geophys. Space Phys., 20:250-265.

Bucher, K., and Frei, M., 1994. Petrogenesis of Metamorphic Rocks: Berlin (Springer-Verlag).

Cannat, M., Bideau, D., and Bougault, H., 1992. Serpentinized peridotites and gabbros in the Mid-Atlantic Ridge axial valley at $15^{\circ} 37^{\prime} \mathrm{N}$ and 165'ㅅ․ Earth Planet. Sci. Lett., 109:87-106.
Cannat, M., Karson, J.A., Miller, D.J., et al., 1995. Proc. ODP, Init. Repts., 153: College Station, TX (Ocean Drilling Program).

Cannat, M., and ODP Shipboard Scientific Party, 1995. Probing the foundation of the Mid-Atlantic Ridge. Eos, 76:129-133.

Christiansen, F.G., 1985. Deformation fabric and microstructures in ophiolitic chromitites and host ultramafics, Sultanate of Oman. Geol. Rundsch., 74:61-76.

Dick, H.J.B., Fisher, R.L., and Bryan, W.B., 1984. Mineralogic variability of the uppermost mantle along mid-ocean ridges. Earth Planet. Sci. Lett., 69:88-106.

Hamlyn, P.R., and Bonatti, E., 1980. Petrology of mantle-derived ultramafics from the Owen fracture zone, Northwest Indian Ocean: implications for the nature of the oceanic upper mantle. Earth Planet. Sci. Lett., 48:6579.

Hofmann, A.W., 1988. Chemical differentiation of the Earth: the relationship between mantle, continental crust, and oceanic crust. Earth Planet. Sci. Lett., 90:297-314.

Ikin, N.P., and Harmon, R.S., 1983. A stable isotope study of serpentinization and metamorphism in the Highland Border suite, Scotland, U.K Geochim. Cosmochim. Acta, 47:153-167.

Ito, E., White, W.M., and Göpel, C., 1987. The O, Sr, Nd and $\mathrm{Pb}$ isotope geochemistry of MORB. Chem. Geol., 62:157-176.

Karson, J.A., and Winters, A.T., 1992. Along-axis variations in tectonic extension and accommodation zones in the MARK area, Mid-Atlantic Ridge $23^{\circ} \mathrm{N}$ latitude: ophiolites and their modern oceanic analogues. In Parsons, L.M., Murton, B.J., and Browning, P. (Eds.), Ophiolites and Their Modern Oceanic Analogues. Geol. Soc. Spec. Publ. London, 60:107-116.

Kretz, R., 1963. Distribution of magnesium and iron between orthopyroxene and calcic pyroxene in natural mineral assemblages. J. Geol., 71:773785 .

, 1982. Transfer and exchange equilibria in a portion of the pyroxene quadrilateral as deduced from natural and experimental data. Geochim. Cosmochim. Acta, 46:411-421.

Kyser, T.K., O'Neil, J.R., and Carmichael, I.S.E., 1982. Genetic relations among basic lavas and ultramafic nodules: evidence from osygen isotope compositions. Contrib. Mineral. Petrol., 81:88-102.

Lindsley, D.H., 1983. Pyroxene thermometry. Am. Mineral., 68:477-493.

Lindsley, D.H., and Dixon, S.A., 1975. Diopside-enstatite equilibria at $850^{\circ}$ to $1,400^{\circ} \mathrm{C}, 5$ to $35 \mathrm{kbar}$. Am. J. Sci., 276:1282-1301.

Margaritz, M., and Taylor, H.P., Jr., 1974. Oxygen and hydrogen isotope studies of serpentinization in the Troodos ophiolite complex, Cyprus. Earth Planet. Sci. Lett., 23:8-14. 
Mattey, D., Lowry, D., and Macpherson, C., 1994. Oxygen isotope composition of mantle peridotite. Earth Planet. Sci. Lett., 128:231-241.

Matthews, A., Goldsmith, J.R., and Clayton, R.N., 1983. Oxygen isotope fractionations involving pyroxenes: the calibration of mineral-pair geothermometers. Geochim. Cosmochim. Acta, 47:631-644.

Michael, P.J., and Bonatti, E., 1985. Peridotite composition from the North Atlantic: regional and tectonic variations and implications for partial melting. Earth Planet. Sci. Lett., 73:91-104.

Miyashiro, A., Shido, F., and Ewing, M., 1969. Composition and origin of serpentinites from the Mid-Atlantic Ridge, $24^{\circ}$ and $30^{\circ} \mathrm{N}$ latitude. Contrib. Mineral. Petrol., 23:117-127.

Morimoto, N., et al., 1989. Nomenclature of pyroxenes. Can. Mineral., 27:143-156.

Muehlenbachs, K., 1976. Oxygen isotope geochemistry of DSDP Leg 34 Basalts. In Yeats, R.S., Hart, S.R., et al., Init. Repts. DSDP, 34: Washington (U.S. Govt. Printing Office), 337-339.

, 1977. Oxygen isotope geochemistry of rocks from DSDP Leg 37. Can. J. Earth Sci., 14:771-776.

, 1978. Oxygen isotope geochemistry of rocks from Leg 46. In Dmitriev, L., Heirtzler, J., et al., Init. Repts. DSDP, 46: Washington (U.S. Govt. Printing Office), $257-258$.

, 1979. The alteration and aging of the basaltic layer of the sea floor: oxygen isotope evidence from DSDP/IPOD Leg 51, 52, and 53. In Donnelly, T., Francheteau, J, Bryan, W., Robinson, P., Flower, M., Salisbury, M., et al., Init. Repts. DSDP, 51, 52, 53: Washington (U.S. Govt. Printing Office), 1159-1167.

Muehlenbachs, K., and Clayton, R.N., 1971. Oxygen isotope ratios of submarine diorites and their constitutent minerals. Can. J. Earth Sci., 8:1591-1595.

1972a. Oxygen isotope studies of fresh and weathered submarine basalts. Can. J. Earth Sci., 9:172-184.

1972b. Oxygen isotope geochemistry of submarine greenstones. Can. J. Earth Sci., 9:471-478.

, 1976. Oxygen isotope composition of the oceanic crust and its bearing on seawater. J. Geophys. Res., 81:4365-4369.

Nicolas, A., Boudier, F., and Bouchez, J.L., 1980. Interpretation of peridotite structures from ophiolitic and oceanic environments. Am. J. Sci., 280:192-210
Obata, M., 1976. The solubility of $\mathrm{Al}_{2} \mathrm{O}_{3}$ in orthopyroxenes in spinel and plagioclase peridotites and spinel pyroxenite. Am. Mineral., 61:804-816.

Peters, T., 1968. Distribution of $\mathrm{Mg}, \mathrm{Fe}, \mathrm{Ca}$ and $\mathrm{Al}$ in coexisting olivine, orthopyroxene and clinopyroxene in the total serpentinite (Davos, Switzerland) and in the Alpine metamorphosed Malenco serpentinite (N. Italy). Contrib. Mineral. Petrol., 18:65-75.

Purdy, G.M., and Detrick, R.S., 1986. Crustal structure of the Mid-Atlantic Ridge at $23^{\circ} \mathrm{N}$ from seismic refraction studies. J. Geophys. Res, 91:3739-3762.

Sachtleben, T., and Seck, H.A., 1981. Chemical control of Al-solubility in orthopyroxene and its implication on pyroxene geothermometry. Contrib. Mineral. Petrol., 78:157-165.

Taylor, H.P., Jr., 1978. Oxygen and hydrogen isotope studies of plutonic granitic rocks. Earth Planet. Sci. Lett., 38:177-210.

Wenner, D.B., and Taylor, H.P., 1973. Oxygen and hydrogen isotope studies of the serpentinization of ultramafic rocks in oceanic environments and continental ophiolitic complexes. Am. J. Sci., 273:202-239.

Wenner, D.B., and Taylor, H.P., Jr., 1971. Temperatures of serpentinization of ultramafic rocks based on ${ }^{16} \mathrm{O} /{ }^{18} \mathrm{O}$ fractionation between coexisting serpentine and magnetite. Contrib. Mineral. Petrol., 32:165-185.

Witt-Eickschen, G., and Seck, H.A., 1991. Solubility of $\mathrm{Ca}$ and $\mathrm{Al}$ in orthopyroxene from spinel peridotite: an improved version of an empirical thermometer. Contrib. Mineral. Petrol., 106:431-439.

Wood, B.J., and Banno, S., 1973. Garnet-orthopyroxene and orthopyroxeneclinopyroxene relationships in simple and complex systems. Contrib. Mineral. Petrol., 42:109-124

Wyllie, P.J., 1981. Plate tectonics and magma genesis. Geol. Rundsch., 70:128-153.

Yui, T.-F., Yeh, W.-H., and Lee, C.W., 1990. A stable isotope study of serpentinization in the Fengtien ophiolite, Taiwan. Geochim. Cosmochim. Acta, 54:1417-1426.

Date of initial receipt: 24 August 1995

Date of acceptance: 27 February 1996

Ms 153SR-018 